CAHIERS DE

NARRATOLOGIE

\section{Cahiers de Narratologie}

Analyse et théorie narratives

$16 \mid 2009$

Images et récits

\title{
Quand le film raconte l'image. Variations cinématographiques autour de La Cène de Léonard de Vinci
}

\section{Valentine Robert}

\section{OpenEdition}

Journals

Édition électronique

URL : http://journals.openedition.org/narratologie/956

DOI : 10.4000/narratologie.956

ISSN : 1765-307X

Éditeur

LIRCES

Référence électronique

Valentine Robert, «Quand le film raconte l'image. Variations cinématographiques autour de La Cène de Léonard de Vinci », Cahiers de Narratologie [En ligne], 16 | 2009, mis en ligne le 25 mai 2009, consulté le 19 avril 2019. URL : http://journals.openedition.org/narratologie/956 ; DOI : 10.4000/narratologie.956

Ce document a été généré automatiquement le 19 avril 2019

Article L.111-1 du Code de la propriété intellectuelle. 


\title{
Quand le film raconte l'image. Variations cinématographiques autour de La Cène de Léonard de Vinci
}

\author{
Valentine Robert
}

1 L'« entrée du film en récit ${ }^{1}$ », pour reprendre les termes de Paul Ricoeur ${ }^{2}$, a été identifiée autour des années 1910 et consiste, comme l'a démontré André Gaudreault ${ }^{3}$, en un changement dans la manière de concevoir le plan cinématographique. Avant 1908-1910, les plans étaient de fait élaborés comme des images autonomes et isolées les unes des autres. Théorisée en termes de "style non-continu " par Tom Gunning ${ }^{4}$, cette acception du plan comme unité expressive semble répondre à ce que Bazin, dans le cadre d'une réflexion qui ne concerne pas le cinéma des premiers temps mais les rapports entre cinéma et peinture, appelle une logique « centripète » de l'image ${ }^{5}$. D'ailleurs, ainsi que l'a montré Jean Giraud, les plans connaissaient à cette époque l'appellation de «tableaux ${ }^{6}$ ». Dès 1908-1910, on commença à concevoir les plans comme des images qui se continuent l'une l'autre, qui sont interdépendantes et se situeraient dès lors dans une polarisation " centrifuge ${ }^{7}$ ", puisqu'elles ne sont pensées que comme des fragments d'espace, de temps et de sens, mais qui dès lors pourront, une fois linéarisées par le montage, agencer un récit. C'est ce décloisonnement des "plans-tableaux ${ }^{8}$ » et leur mise en continuité qui a ainsi été établie comme déterminante de la « narrativisation » du cinéma.

Or, si, conformément à ce changement dans la manière de penser l'image cinématographique, Gaudreault recense "au tournant des années dix ", de nombreuses "tentatives d'annulation de l'autonomie des plans ${ }^{9}$ ", je voudrais dans cet article faire état de tentatives " inverses ", consistant à réaffirmer l'autonomie de certaines images, à réintroduire, au sein du continuum des plans filmiques, des plans-tableaux. Car une telle pratique existe, s'étend bien au-delà des années dix, et présente même une récurrence significative au sein d'une certaine catégorie de films «narratifs». Je propose en effet d'analyser la manière dont le plan-tableau, qui a dans l'historiographie été posé comme le repoussoir du récit filmique, va refaire irruption au sein de certains films, non pour en 
"arrêter» le récit, mais au contraire pour intéragir avec le montage narratif et l'amplifier de modalités nouvelles, héritées de la peinture ou de certaines pratiques théâtrales comme le tableau vivant. Le cadre de cette analyse est donné par des films mettant en scène le Christ. De fait, cette figure iconographique par excellence porte avec elle quelque vingt siècles de représentations, dont les cinéastes ne peuvent faire l'économie. Et certains «modèles " plastiques incontournables - parmi lesquels figurent au premier chef des "peintures d'histoire ${ }^{10}$ ", autrement dit des modèles également narratifs - vont parfois ressurgir au sein des plans filmiques, en réintroduisant une lecture d'image tabulaire au sein du régime visuel linéaire ${ }^{11}$.

3 Les films des premiers temps qui représentent Jésus - lesdites " Passions filmées ", très nombreuses et populaires entre 1897 et 1908 - sont d'ailleurs reconnus par André Gaudreault, Noël Burch, et la plupart des théoriciens du cinéma des premiers temps, comme des films emblématiques de la «transition» entre deux «modes de représentation " cinématographiques ${ }^{12}$, en vertu de la parfaite ambivalence de leurs " plans ", qui sont travaillés et déchiffrés dans une logique à la fois tabulaire et linéaire. Chaque prise de vue des Passions filmées est en effet construite à la manière d'une scène, englobant une unité de temps, d'espace et d'action, et conçue comme un tableau au sens propre, puisque la tradition iconographique - tant picturale que théâtrale - en a codifié la composition à tel point que les protagonistes viennent souvent « prendre la pose » au sein $\mathrm{du}$ "plan", qui s'immobilise dès lors en tableau vivant ${ }^{13}$. Par exemple, le baptême du Christ mis en scène - et «en pose »- dans la Passion Pathé de 1905 [fig. 1], présente une composition calquée sur des codifications iconographiques qui traversent les frontières (identiques dans la sphère orthodoxe et occidentale), les siècles (perdurant du IV ${ }^{e}$ siècle à l'époque moderne) et les supports (apparaissant à la fois sur les mosaïques antiques, les icônes, les groupes sculptés des fonts baptismaux, les peintures d'histoire, gravures et estampes populaires ${ }^{14}$ [fig.2-3]). Mais si les «plans» de ces Passions des premiers temps sont travaillés comme des scènes iconographiques autonomes, ils ne visent pas moins à être appréhendés, grâce au référent qu'est le « récit » évangélique, dans une continuité narrative, en différentes « phases » successives de la vie du Christ ${ }^{15}$.

[fig. 1]

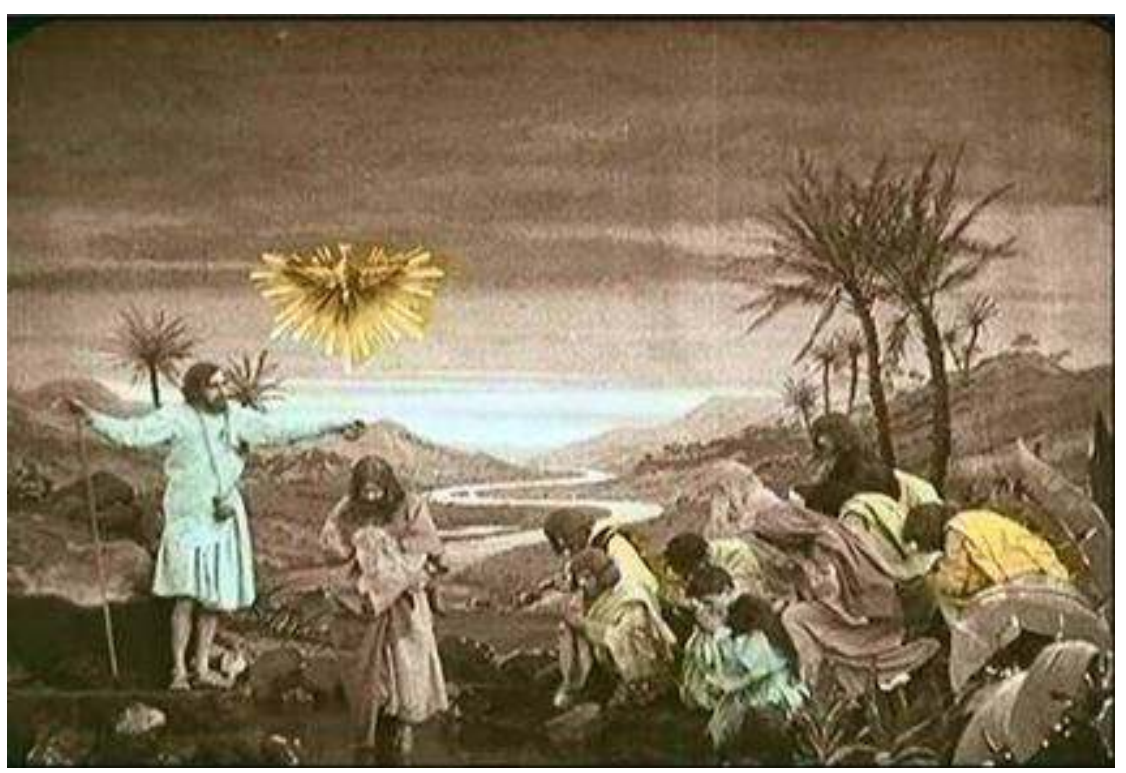

La Vie et la Passion de Notre Seigneur Jésus-Christ (Zecca \& Nonguet, 1905) [Tous droits réservés] 
[fig. 2]

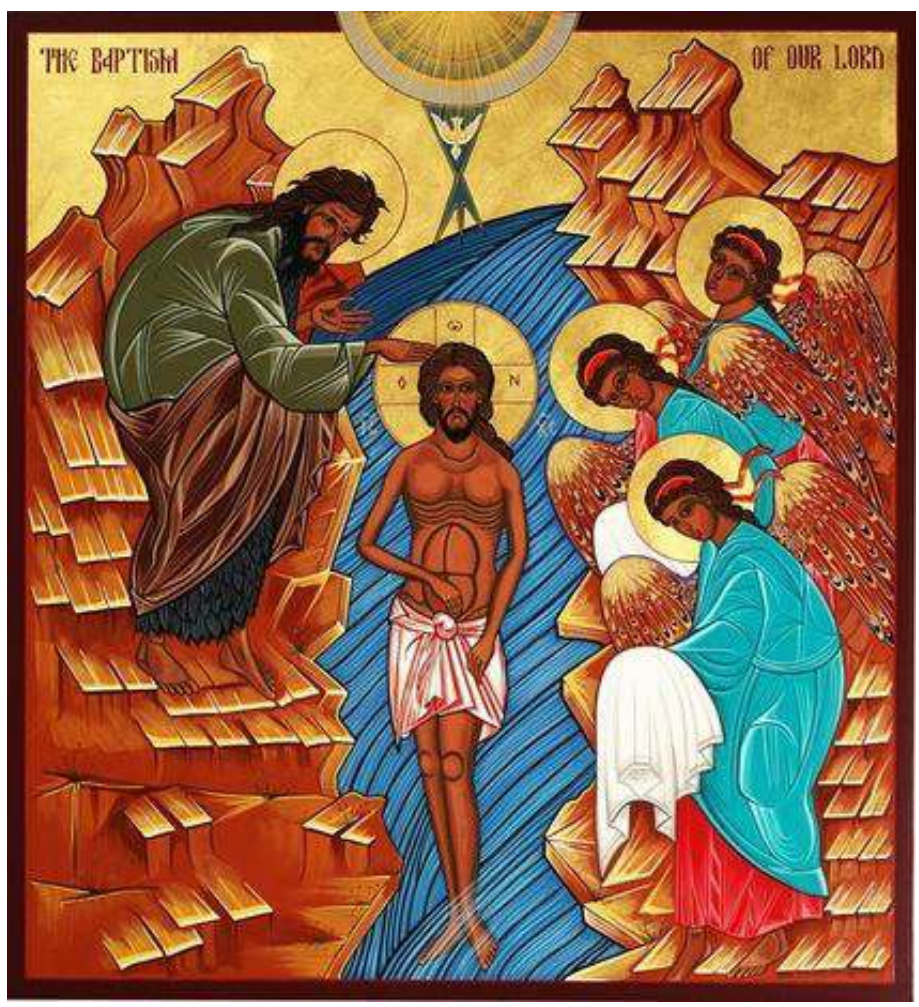

Canevas de l'icône de la théophanie [Tous droits réservés]

[fig. 3]

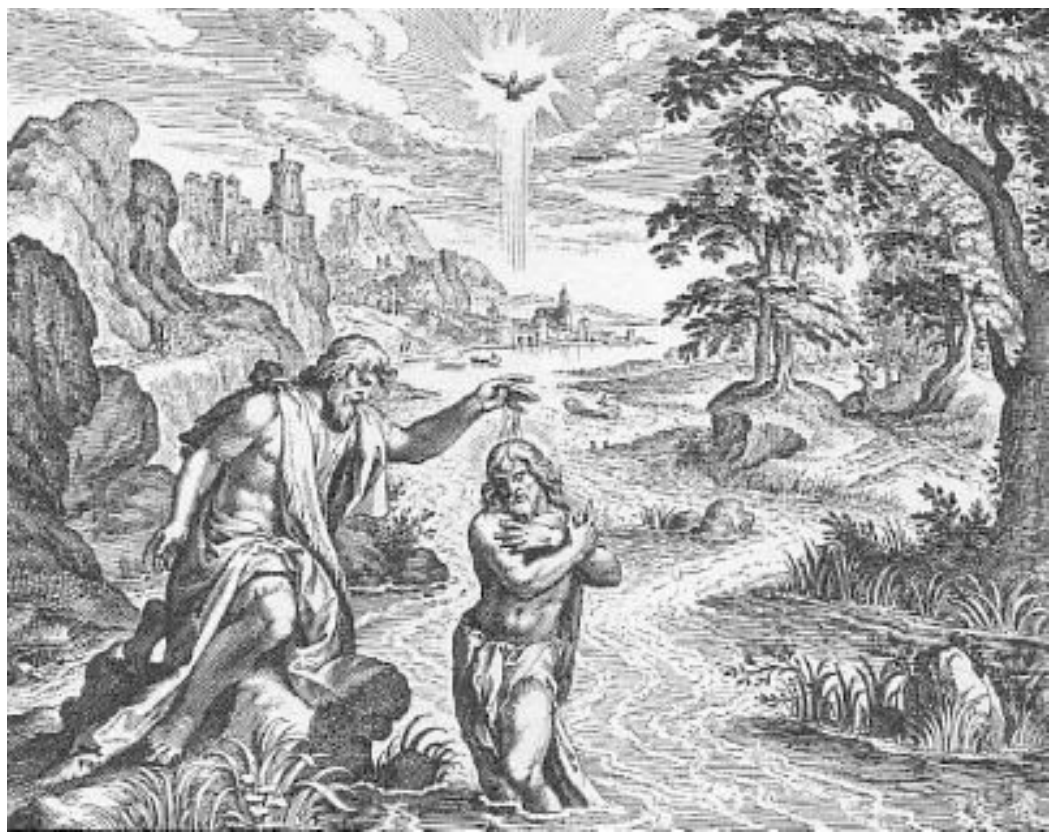

L'Ainé (Gravure. Matthaeus Merian, 1625-1630) [Tous droits réservés]

4 Cette double logique de l'image (que Burch dirait mi «autarcique », mi « linéarisée ») s'inscrit d'ailleurs dans la tradition scénographique des Passions, puisque ces spectacles 
liturgiques - théâtraux, mais aussi picturaux ou sculpturaux - ont la spécificité de fonctionner en «stations ». La représentation des Passions est de fait historiquement prise entre la fixité et le mouvement, disséminée le long du chemin de croix en plusieurs tableaux ou tréteaux devant lesquels les spectateurs font « halte». Ce dispositif, dont la vogue est remarquable à la fin du XIXe siècle, induit donc une double perception des images christiques qui «mènent l'une à l'autre » tout en sollicitant chacune une lecture autonome, un temps de recueillement et d'« isolement ». Ce n'est donc pas un hasard si les ambivalentes «stations" de la Passion deviennent un sujet privilégié du plan cinématographique, à l'heure de son développement «intermédiaire", entre le plantableau et le " plan-segment ${ }^{16}$ ».

5 Mais cette ambivalence du plan (et plus particulièrement du plan christique) n'est pas uniquement le fruit d'une transition cinématographique historique, puisqu'elle s'actualise encore, notamment, dans l'une des dernières Passions filmées (ou plus précisément «filmiques ») en date : La Passion du Christ réalisée en 2004 par Mel Gibson. En effet, après la déposition du Christ survient le plan d'une pietà qui subitement « se fait tableau » [fig. 4].

Une rupture dans la « linéarité » des plans s'établit, non seulement par l'immobilisation de la Vierge, dont la rigidité de la pose est soulignée par le contraste de l'agitation du vent et du lent mouvement de caméra, mais aussi et surtout par un geste de Marie. En effet, le passage du plan au tableau survient au moment où, en un gros plan, le regard de la Vierge se détache du corps mort de son fils, pour venir, lentement, avec tout le poids que représente cet acte, s'adresser au spectateur. Ce regard-caméra brise les codes classiques $d u$ récit cinématographique, qui ont pour principe d'«immerger» le spectateur dans l'histoire, de faire oublier le dispositif afin que la narration semble «se raconter d'elle-même ». En transgressant ici la frontière du "quatrième mur " matérialisé par l'écran, Marie fait «exploser » le sens de ce plan - et de l'ensemble du film, en l'amplifiant d'une logique picturale. Car une multitude de peintures - surtout les crucifixions, mais également certaines pietà, à l'instar de celle de Bouguereau [fig. 5] présentent la Vierge comme l'admoniteur albertien ${ }^{17}$, autrement dit comme le personnage qui adresse le tableau au spectateur par le regard. La Vierge Marie appelle donc à une lecture "tabulaire » du plan, une appréhension frontale et fixe de l'image, comme le souligne explicitement le mouvement de caméra. La «force tabulaire » de ce plan émane en effet aussi du lent travelling arrière qui fait glisser la caméra sur l'axe transversal reliant l'image à son spectateur. Ce mouvement retrouve la logique visuelle des films des premiers temps, où les raccords cut-in (dans l'axe) dynamisaient l'image en profondeur, maintenant la frontalité du cadrage par rapport à l'objet, avec les implications centripètes, théâtrales et picturales qu'André Chaperon a montrées ${ }^{18}$. 
[fig. 4]

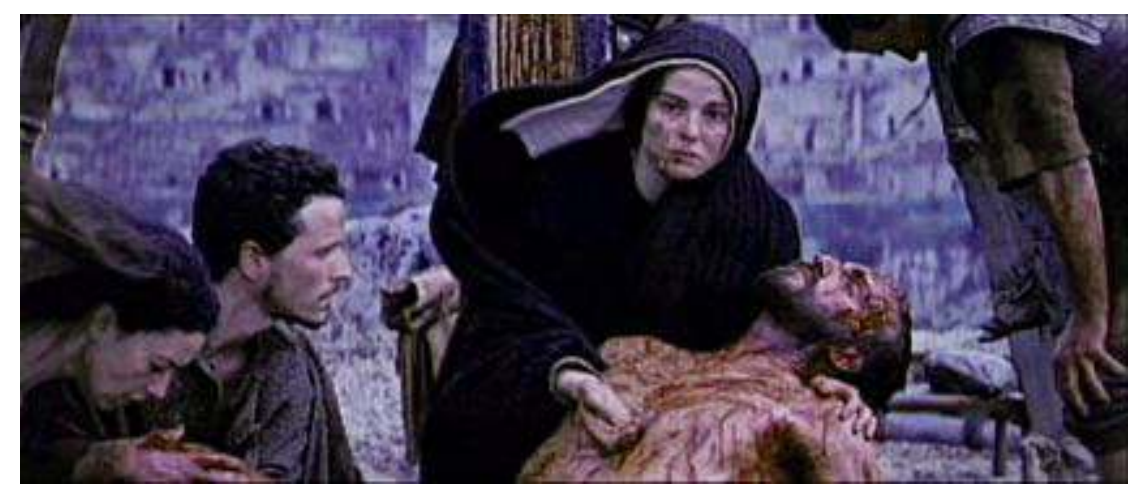

The Passion of the Christ (Mel Gibson, 2004) [Tous droits réservés]

[fig. 5]

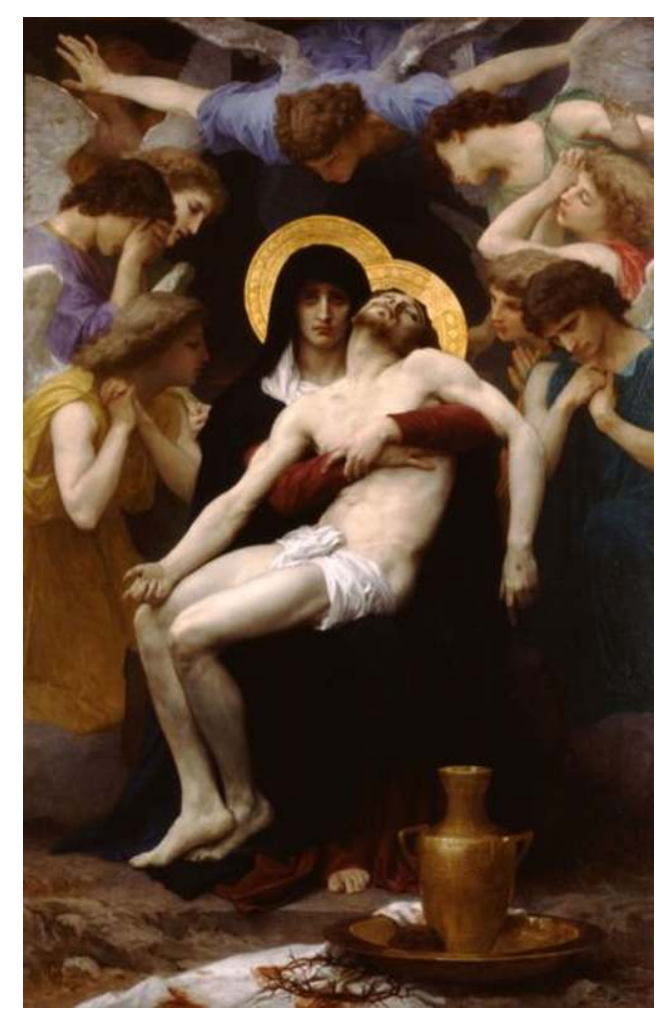

Piéta (William Adolphe Bouguereau, 1876) [Tous droits réservés]

7 Ce mouvement ne "s'achèvera " pas, toutefois, n'immobilisant jamais le plan en image fixe, puisqu'un fondu au noir vient «effacer » le «tableau » attendu. L'image disparaît avant que le cadrage, parti d'un gros plan sur Marie et élargi progressivement au fil du travelling arrière, n'ait englobé la totalité de la composition, suivant les conventions iconographiques traditionnelles. Et plusieurs éléments contribuent à «fondre » cette image dans la logique continue des plans qui la précèdent: la musique chorale, qui harmonise et unifie la succession des images, la mise en contexte de cette posture au sein de l'action narrative globale (la déposition), enfin l'« annonce » du glissement vers une logique visuelle contemplative par l'occurrence répétée d'un "contrechamp » sur les [futures] reliques de la Passion, regroupées et «composées» selon les schémas 
iconographiques traditionnels [fig. 6]. Le plan ne s'autonomise donc jamais complètement en tableau, mais demeure en tension entre les deux régimes.

[fig. 6]

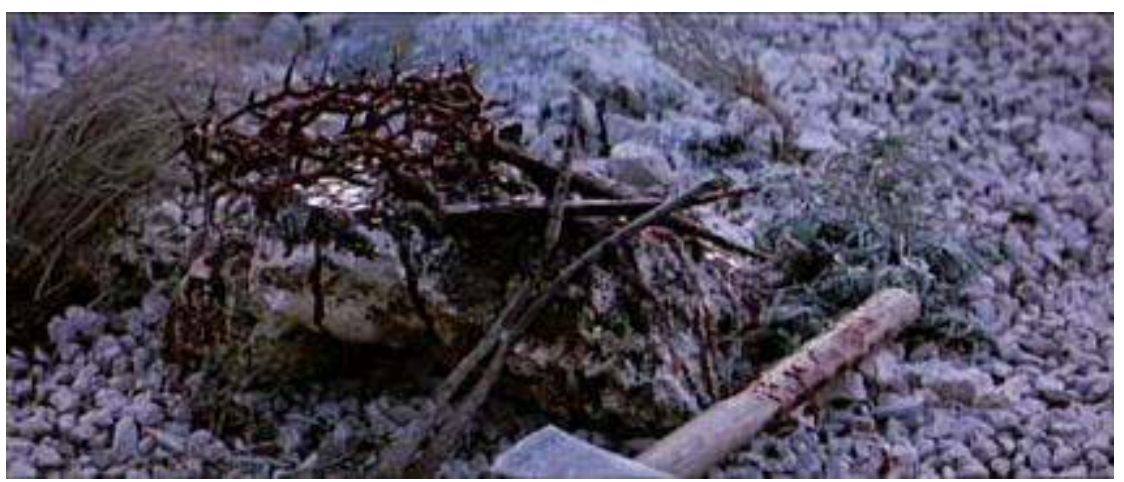

The Passion of the Christ (Mel Gibson, 2004) [Tous droits réservés]

8 Les effets de sens de ce plan-tableau sont tout d'abord émotionnels. Rappelons que la scène de la pietà, qui incarne le climax passionnel de l'histoire sainte, n'a pas d'existence dans le récit évangélique, mais est un thème uniquement iconographique ; autrement dit, elle ne prend sens que par l'image, par cette intensité dramatique, ce «mouvement de l'âme", qui lui serait propre ${ }^{19}$. Le fait de "suspendre» le récit en cette composition ne fait que renforcer l'intensité du moment. Or, cela renoue avec l'origine-même des tableaux vivants. En effet, tel que Diderot le définit, le «tableau » intervenait au sein des pièces de théatre pour en "suspendre » l'action lorsqu'elle prenait une ampleur paroxystique, pour souligner et immortaliser l'émoi dramatique sur un effet plastique. C'était le «clou » du spectacle, qui condensait l'expressivité des acteurs par ces stases spectaculaires appelées alors "tableaux dramatiques", non sans résonnance émotionnelle ${ }^{20}$.

9 Le regard caméra qui « saisit » le spectateur ne sert pas uniquement à décupler la portée passionnelle du plan-tableau en engageant le spectateur à participer au recueillement sur le corps défunt. Ce plan-tableau devient également le moyen d'un message théologique, car la Vierge donne un « avertissement » non seulement visuel mais également moral au spectateur, appelant sa compassion mais aussi sa culpabilité et, à terme, sa confession, comme l'ensemble des représentations montrant le Christ souffrant ou mort ${ }^{21}$. En retrouvant une élaboration sémiotique tabulaire, et en se résorbant par fondu au noir dans un imaginaire visuel canonique, ce plan-tableau dramatique et (presque) final ${ }^{22}$ vient rattacher l'ensemble du film à la tradition iconographique. «Vectorisé » non plus dans une linéarité narrative mais dans une axialité spectatorielle, le sens du film se décuple en réactivant un message symbolique, théologique et artistique déployé en cinq siècles de représentations.

Cène

Des Passions filmées des premiers temps à la Passion du Christ de 2004, les exemples d'une double élaboration du plan-tableau n'ont cessé de se multiplier au sein des films thématisant cette figure iconographique par excellence. Dans cette étude, je me concentrerai sur un cas précis et récurrent de plan-tableau christique au cinéma: les occurrences de la composition de la Cène de Léonard de Vinci ${ }^{23}$ [fig. 7]. 
11 Cette fresque est en quelque sorte le symbole de l'iconographie, du modèle visuel codifié (voire «codé»). A tel point que l'historien d'art Léo Steinberg la présente comme « l'image narrative la plus copiée, adaptée, détournée et satirisée » qui ait jamais existé ${ }^{4}$. Et le cinéma a pris part à ces imitations, qui, depuis une copie gravée d'à peine deux ans postérieure à la fresque ${ }^{25}$, jusqu'à la publicité de Marithé et Girbaud en $2005^{26}$, en passant par des tatouages ${ }^{27}$, des parodies en tous genres ${ }^{28}$ aussi bien que par des œuvres contemporaines engagées ${ }^{29}$ et des installations postmodernes ${ }^{30}$, cultivent la composition de Vinci comme une inépuisable matrice formelle et symbolique, voire narrative, si l'on pense aux innombrables lectures et interprétations que la fresque a suscitées, et dont la plus célèbre reste bien sûr celle proposée par le $D a$ Vinci Code ${ }^{31}$, qui va jusqu'à faire du tableau le révélateur d'une nouvelle histoire évangélique, où Jésus consacre, en guise de Graal, sa femme porteuse de sa descendance (son "sang »). Je tenterai ici de dégager les implications intermédiales et narratives qu'entraîne l'occurrence de cette composition tabulaire exemplaire au sein du plan cinématographique.

\section{[fig. 7]}

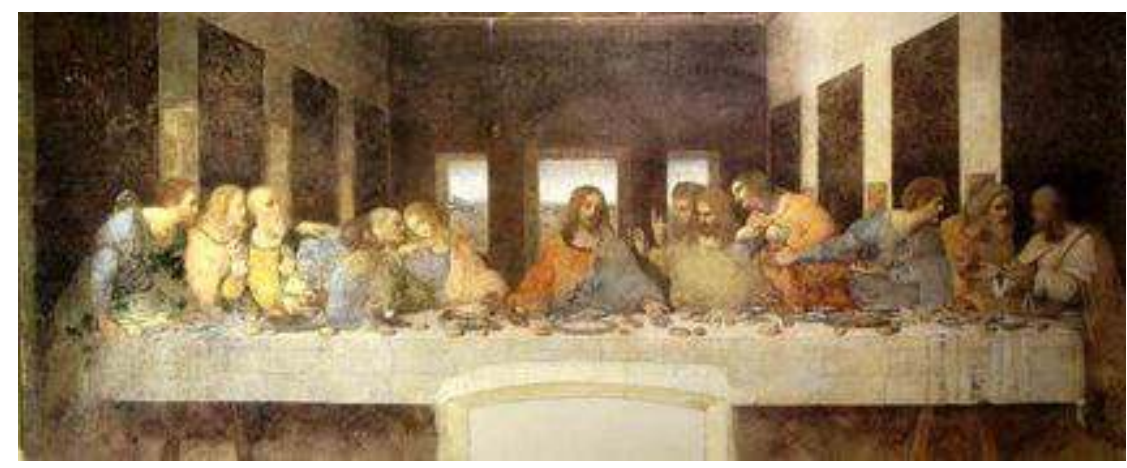

La Cène (Léonard de Vinci, 1494-1498) [Tous droits réservés]

Pour commencer ce parcours à travers les apparitions filmiques du tableau de Vinci, je me concentrerai sur Christus (Giulio Antamoro, 1914-1916), soit l'une des - si ce n'est la plus ancienne appropriation cinématographique de la fresque. Il faut dire que le film a été créé dans les années dix, qu'il fait donc lui-même partie de la période de «transition » entre les deux conceptions du film décrite par Gaudreault, justifiant donc historiquement cette ambivalence entre tableau et continuité des plans. De plus, ce film a pour principe même de cultiver la référence picturale, en reprenant sur le mode du tableau vivant des compositions connues, les annonçant par des intertitres et allant même jusqu'à faire surgir un cadre pictural pour concrètement « encadrer » le plan (à l'instar de celui citant 1 'Annonciation de Fra Angelico).

13 La séquence du film consacrée à la Sainte Cène s'ouvre sur un intertitre annonçant que La table est mise par les anges. La « mise en film » (et surtout la mise en " plans » filmiques) est donc annoncée directement comme une narrativisation de la composition de Vinci, inscrite dans une temporalité qui montre l'« avant » du tableau. Et les anges ne font pas que préparer le décor en mettant la table : ils préparent également la symbolique de la (s)cène, en prenant déjà la posture (mains croisées sur la poitrine) des futurs communiants chrétiens, et en allant s'agenouiller côte à côte, de telle sorte à former une grande croix face à la table où s'assoiront les saints, avant de disparaître "miraculeusement». Ils annoncent ainsi tous les enjeux narratifs et symboliques de l'eucharistie, présageant de la crucifixion comme "prochaine étape annoncée » de 
l'histoire sainte, et de la communion comme une voie vers le Christ, vers le Salut. Sur un plan formel, ils indiquent également l'importance esthétique et signifiante de la disposition et de la « pose » des protagonistes dans la séquence.

Le Christ et les apôtres entrent dans le plan par la droite - il ne s'agit pas, comme avec les anges, d'une apparition générée miraculeusement au sein de l'image. Le plan n'est plus pensé comme un espace clos "autarcique", puisque l'une des tapisseries sombres conçues par Léonard en bordure du décor pour rythmer formellement l'espace, le « recentrer » et l'autonomiser, est transformée dans le film en une porte ouvrant sur un autre espace, attenant, un hors-champ duquel proviennent les figures sacrées. Le tableau " centripète » est transformé ici en un plan filmique "centrifuge ", en même temps que sa profondeur tridimensionnelle se manifeste de manière ambiguë, comme l'a montré Alain Boillat :

[C]ette confusion entre porte et tenture révèle le «clivage entre la présence du point de fuite [...] et une représentation tout en surface " qu'Anne-Françoise Lesuisse (Du film noir au noir, DeBoeck, Bruxelles, 2002, p. 64) constate à propos des films des premiers temps réalisés grâce à la technique de la toile peinte ; cet aspect [étant] particulièrement intéressant ici, dans la mesure où c'est paradoxalement la construction géométrique fortement perspectiviste élaborée par le peintre qui confère sa profondeur à l'image filmique ${ }^{32}$.

La composition du tableau de Léonard survient soudain, immobilisée en tableau vivant [fig. 8], encadrée par deux intertitres qui l'isolent des autres plans et de tout mouvement, tant visuel que narratif. Rien en effet ne justifie la composition, aucune causalité ni temporalité n'est donnée à la gestuelle étrange et expressive des apôtres (l'intertitre annonçant Le Cénacle de Leonard de Vinci apparaît alors que les personnages ne se sont pas même assis). La disposition picturale est simplement citée, « appliquée » à ce plan-tableau muet, immobile, et autonome. A ce stade, la seule signification visible de cette mise en scène réside dans la volonté affichée de chercher à légitimer le cinéma comme un art (en cette période où il n'est pas reconnu comme tel), et surtout de souligner sa parenté avec la « grande peinture » d'histoire.

Mais le mouvement (et avec lui le récit) va reprendre dès le plan qui suit l'intertitre " clôturant». Ce cadrage, rapproché autour de la figure christique, révèle un double mouvement narratif, puisqu'il montre en un même plan le partage du pain et l'annonce de la trahison de Judas : on y voit le Christ, après avoir distribué le pain à Judas, chercher le contact avec son disciple, mais le " traitre » se défile à l'accolade de Jésus qui tourne la tête d'un air entendu et triste. Or, la Cène de Léonard de Vinci est précisément le lieu d'un débat des historiens d'art quant à savoir si elle représente l'instant où le Christ annonce que l'un des disciples le trahira, ou le moment de l'institution de l'eucharistie; et l'une des interprétations les plus établies consiste à dire que la peinture condense ces deux moments dans un " instant prégnant ${ }^{33}$ ». Les deux temps de l'instant prégnant sont bel et bien restitués dans ce film : l'image filmique se réapproprie, en «traduisant » selon ses propres moyens narratifs que sont la temporalité et le jeu d'acteur, le récit condensé dans la fresque. 
[fig. 8]

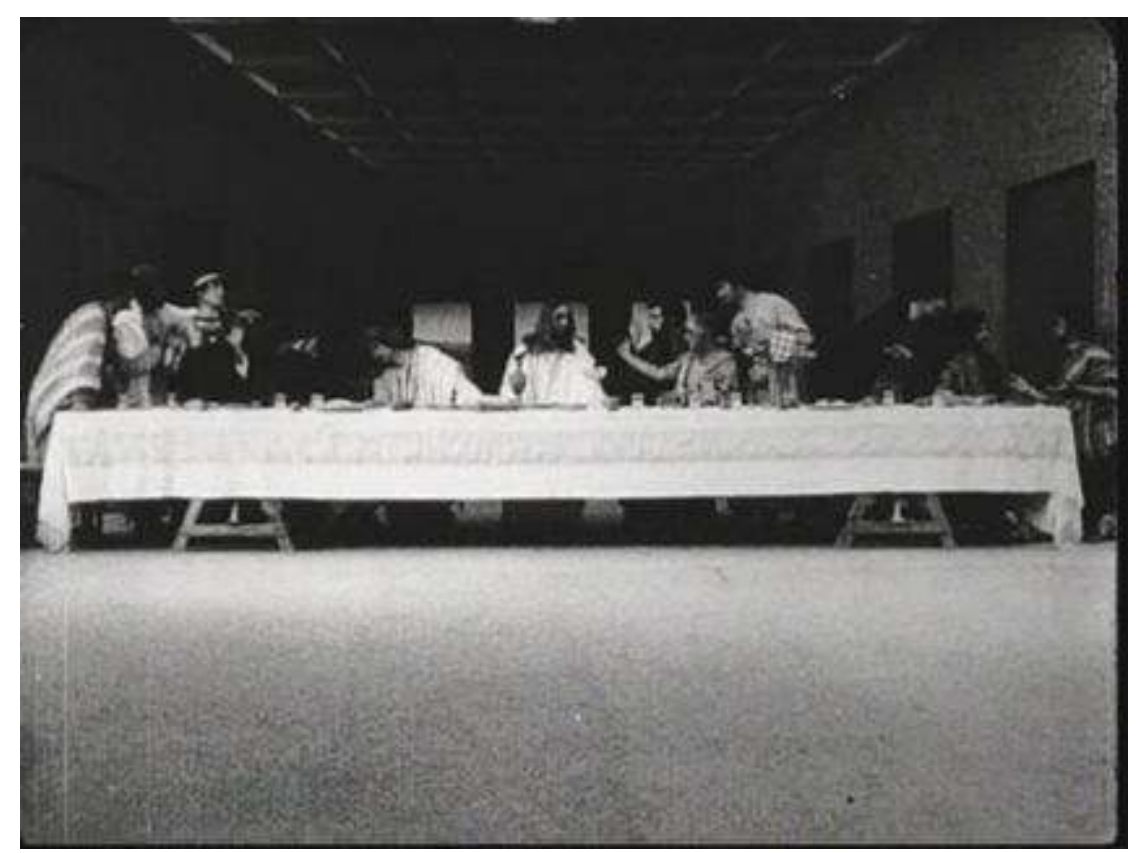

Christus (Giulio Antamoro, 1914-1918) [Tous droits réservés]

Et elle le mène plus loin, puisque le plan se ré-immobilisera en tableau à la fin de la séquence, lorsque Jésus lève le graal vers le ciel et que la colombe du Saint-Esprit apparaît [fig. 9]. L'immobilité est certes nécessaire pour le trucage (la surimpression) qui permet l'«apparition divine ", toutefois la pose du Christ et des apôtres se prolonge pendant quelque vingt secondes. Or, si le premier tableau vivant apparaissait selon une composition et une logique picturale, n'ayant ni mouvement, ni « avant » ni « après », ce dernier tableau vivant présente des caractéristiques cinématographiques :

- il n'est pas présenté comme l'imitation d'un tableau, au contraire, l'intérêt premier de sa composition réside dans un effet visuel de trucage filmique ;

- il n'est pas séparé des autres plans par des intertitres, mais est très fortement inscrit dans la continuité du film, puisqu'un mouvement continu très lent nous permet de voir toutes les phases du geste qui amène cette pose, et puisque ce plan va même être redécoupé par un plan rapproché, suivant une logique de montage cinématographique (celle du cut-in décrite plus haut comme plus « tabulaire » que linéaire »);

- enfin, si le tableau de Léonard apparaissait dépourvu de signification, ici, le tableau scelle le sens narratif et symbolique de la séquence, en explicitant le destin ultime du sacrifice annoncé.

Ce dernier tableau vivant est donc travaillé suivant la tradition dramatique du XVIIIe siècle, en étant mis au service d'un récit dont il accentue le poids, portant le potentiel narratif à son «comble ${ }^{34}$ ». Contrairement au premier plan-tableau qui était essentiellement voué au jeu artistique de références et de tensions intermédiales, telles que les pratiques dix-neuviémistes mondaines du tableau vivant l'ont cultivé, Christus montre ainsi que le plan cinématographique a réactivé et a développé une pratique du tableau vivant d'«avant" son autonomisation formelle du XIXe siècle ${ }^{35}$, et plus globalement a joué d'une référence au tableau pour le "déployer narrativement», en « linéarisant » sa temporalité et son espace dans une suite interdépendante de plans. 
[fig. 9]

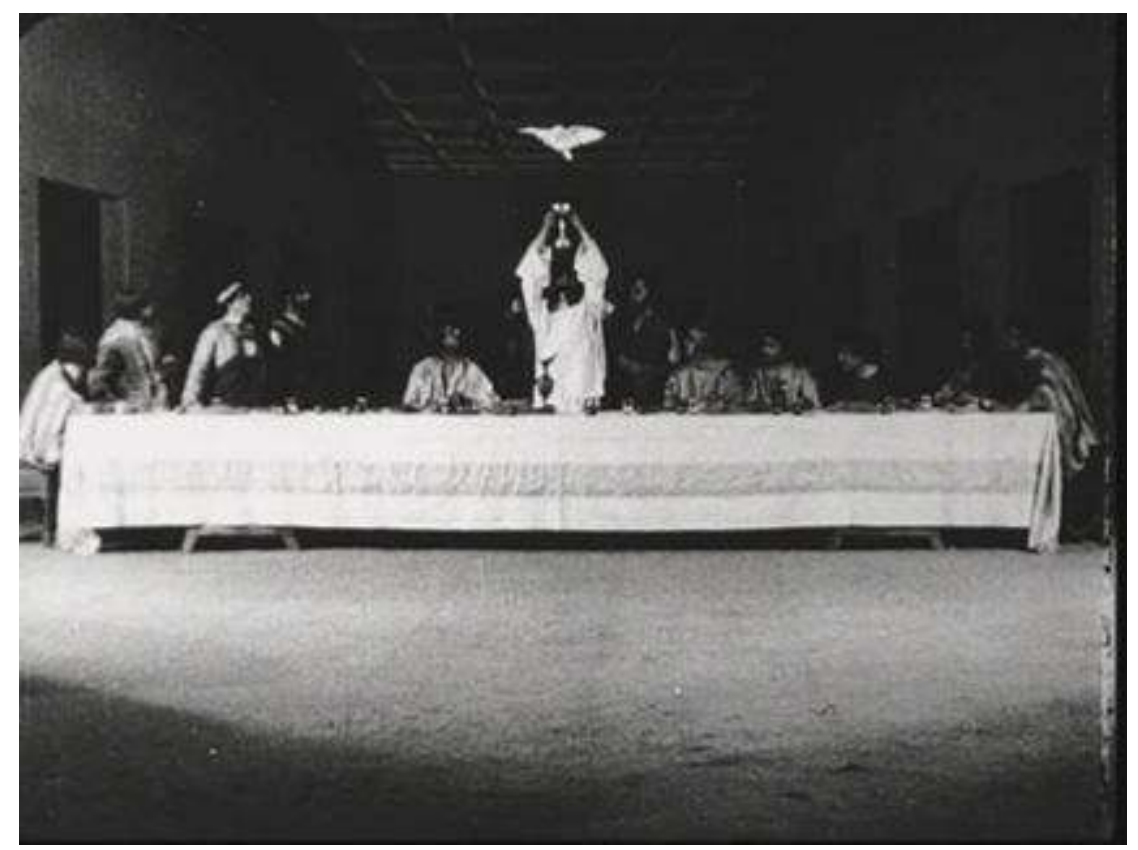

Christus (Giulio Antamoro, 1914-1918) [Tous droits réservés]

19 En tant que modèle iconographique, La Cène de Léonard de Vinci a suscité des imitations cinématographiques rigoureusement "littérales ", comme le tableau vivant purement citationnel de Jesucristo y Maria Magdalena (Miguel Contreras Torres, 1946) : la séquence du dernier repas s'y ouvre en effet sur le tableau léonardien maintenu en pose pendant huit secondes [fig. 10] avant que les acteurs ne se mettent en mouvement et que le Christ ne prenne la parole pour instituer traditionnellement l'eucharistie, la scène se continuant dans un régime parfaitement linéaire, fait de nombreux plans rapprochés montés en continuité, et sans justification aucune du tableau vivant. 
[fig. 10]

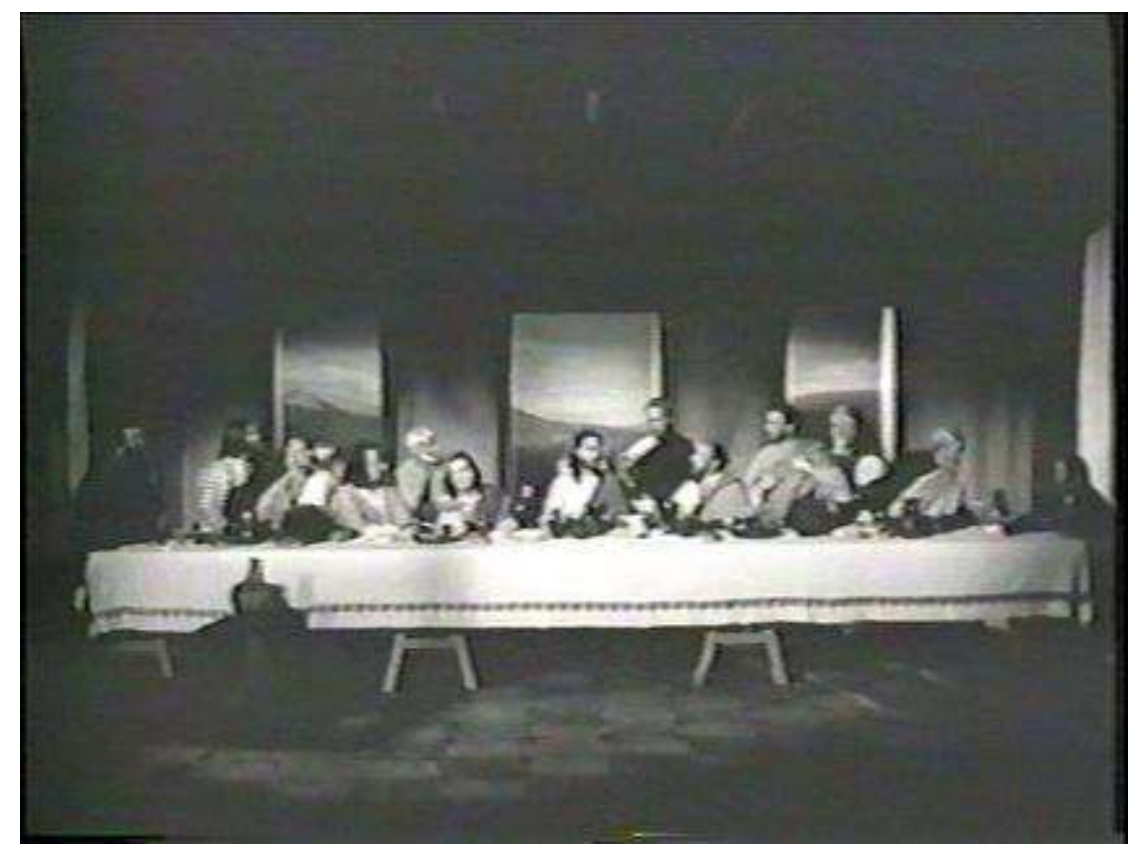

Jesuchristo y Maria Magadalena (Miguel Contreras Torres, 1946) [Tous droits réservés]

Mais l'imitation de cette peinture au cinéma peut aussi être le lieu d'un véritable "paragone ", c'est-à-dire d'un discours plastique réflexif sur la comparaison et la concurrence des arts, comme l'a suggéré Alain Masson en parlant d'« "imitation" au sens que l'ancienne rhétorique donnait à ce mot: il s'agit de la mise en rapport, évidente et significative, de deux langages différents, et ici de deux arts différents ${ }^{36}$ ». Ben Hur, a tale of the Christ (Fred Niblo, 1925) donne le meilleur exemple d'une imitatio cinématographique du tableau de Vinci, la référence à la célébrissime fresque y révélant un discours sur l'iconographie et sur le rapport entre cinéma et peinture.

En effet, le film produit un tableau vivant du Cenacolo, mais en en bousculant la composition : conformément à une convention iconographique florentine concurrente qui trouve notamment chez Domenico Ghirlandaio, soit le maître de Léonard lui-même, de nombreux exemples emblématiques ${ }^{37}$ - Judas est déplacé de l'autre côté de la table. Et ce plan filmique enfreint la composition léonardesque en allant jusqu'à positionner le traître en plein centre, de sorte à ce qu'il cache le Christ dont on ne voit plus que l'auréole et les bras [fig.11]. Cette transgression iconographique est loin d'être gratuite, puisqu'elle répond au principe visuel du film qui consiste à dissimuler le visage de Jésus à l'écran. Jamais le film de Niblo ne personnalise l'acteur jouant le Christ, il n'en révèle que la silhouette, le dos, les bras ou l'aura, en rejetant ses traits hors-champ, bord-cadre ou en les dissimulant derrière un avant-plan comme celui incarné ici par Judas. Le renouvellement de la composition "canonique " de Léonard vient donc appuyer la rupture iconographique amenée par le film, et revendique ce procédé cinématographique comme un nouveau « code » de représentation. Or l'histoire du cinéma donnera raison à cette ambition, puisque jusqu'en 1960, la majorité des films représentant Jésus suivront comme une règle ce principe consistant à cacher le visage du Christ au spectateur, au point que la censure anglaise l'érige en « $\operatorname{loi}^{38}$ ». Si Ben Hur fait de son plan un tableau, 
c'est donc pour proclamer un « relais » entre peinture et cinéma, et ériger le médium filmique en moyen artistique d'une nouvelle iconographie.

\section{[fig.11]}

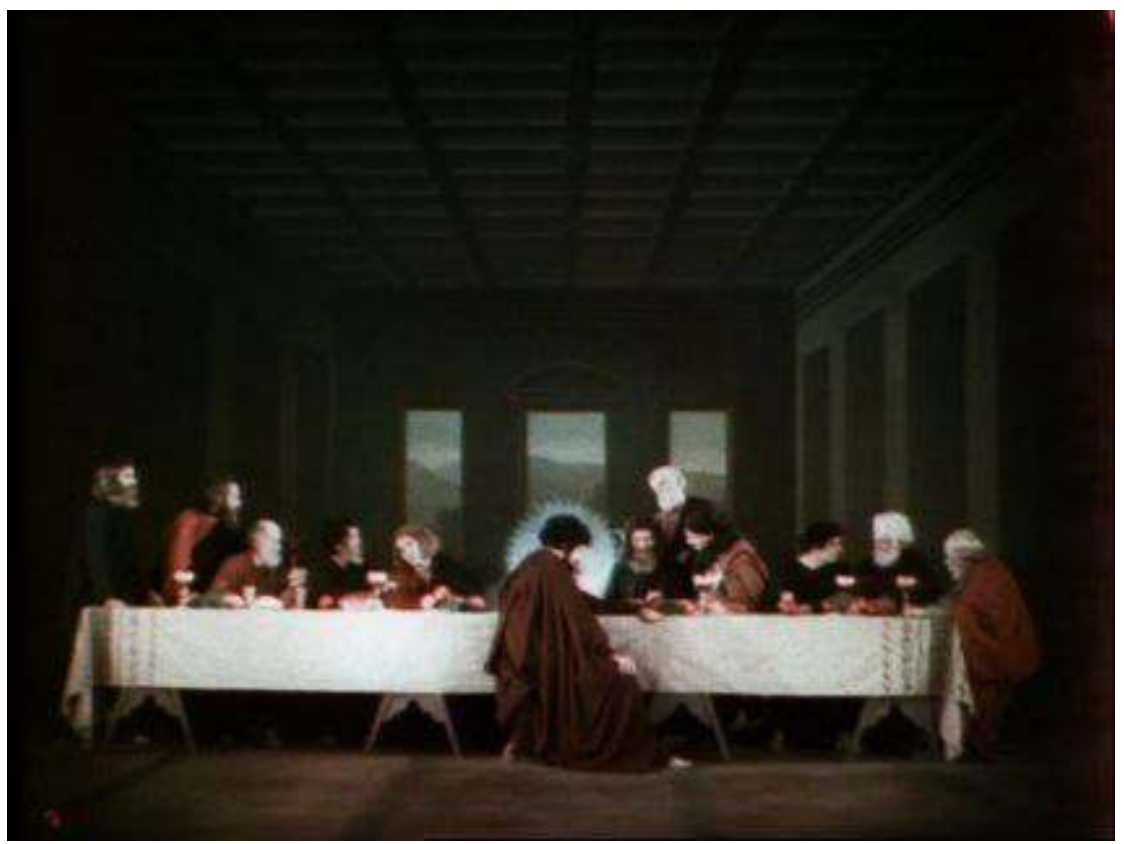

Ben Hur (Fred Niblo, 1925) [Tous droits réservés]

Cette manière de calquer le plan filmique sur le tableau de Vinci en ménageant des écarts, afin de revendiquer un renouveau de l'iconographie par le cinéma, a également été travaillée dans Jesus Christ Superstar (Norman Jewison, 1973) [fig. 12], comme en témoigne son réalisateur :

J'ai fait pousser de l'herbe à cet endroit expressément pour cette scène; je voulais que la Sainte Cène ait lieu dehors, même si nous sommes tous influencés par Léonard de Vinci. La plupart d'entre nous imaginent la cène comme l'a représentée Léonard de Vinci dans son tableau que l'on voit depuis l'enfance, dans les musées, dans des calendriers ou sur les murs des églises, n'importe où. Mais je voulais la mettre en scène dehors, et je les ai fait asseoir par terre parce que c'est comme ça que l'on mangeait à l'époque ${ }^{39}$.

La Cène de Vinci est donc envisagée comme l'« emblème » de la référence iconographique, et si Jewison en reprend le canevas, c'est moins pour exprimer cet héritage que, paradoxalement, pour marquer la différence entre son film et la tradition. Il défend la dissemblance - bien plus que la similitude - entre son plan et le fameux tableau, en vantant les changements amenés au décor (devenu un extérieur verdoyant) et aux postures (assises par terre). L'argument de réalisme historique évoqué en guise de justification de ces « décalages » semble cependant absurde au vu du film qui cultive des jeux d'anachronisme incessants, et du plan qui s'immobilise en tableau vivant de manière purement artificielle et injustifiée. Si la composition de Léonard est retravaillée dans ce film musical (et non historique), c'est bien en tant que modèle plastique et chorégraphique. Mais l'objectif du film reste celui de « dépasser » la peinture, d'en faire la matrice d'une danse et d'un récit déployés grâce aux potentialités propres à l'image en mouvement. 
[fig. 12]

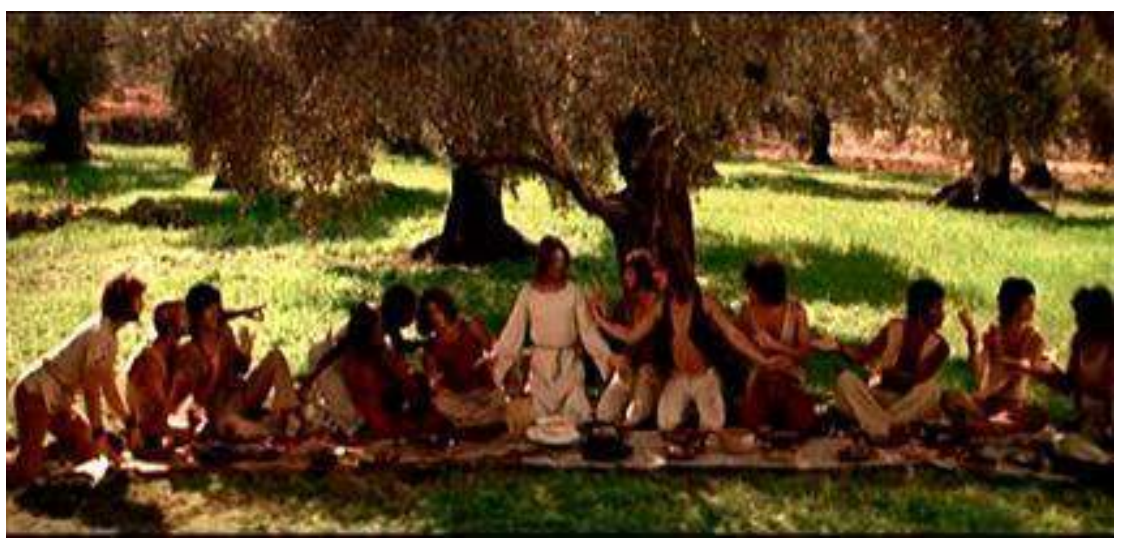

Jesus Christ Superstar (Norman Jewison, 1973) [Tous droits réservés]

Le film Quo Vadis (Mervyn LeRoy, 1951) fait également apparaître la Cène de Vinci en plantableau et problématise sa signification narrative essentiellement dans son rapport à la parole. L'image apparaît au sein du discours de Pierre, narrateur intradiégétique qui fait aux premiers chrétiens le récit de la vie de Jésus telle qu'il en a été le témoin. Au lieu de se «fondre » en un flashback, la voix de Pierre - qui narre le dialogue échangé durant la cène entre lui-même, jurant fidélité à son maitre, et le Christ, refusant de croire ce serment et annonçant le reniement de son plus fidèle disciple - perdure « over » tandis qu'un fondu fait apparaitre l'image de la Cène "figée » en un tableau vivant (sans toutefois que les poses ne soient absolument exactes) [fig. 13]. Ce mode est des plus intéressants car il renoue avec une tradition de la représentation de la vie du Christ antérieure au cinéma, remontant aux bibles illustrées qui, depuis la Biblia Pauperum médiévale, en passant par les œuvres monumentales de Gustave Doré et James Tissot, ont modélisé une imagerie évangélique en vignettes emblématiques, séquentialisées au fil d'un récit verbal autonome, ainsi qu'aux méthodes de catéchisme en images ${ }^{40}$, qui eurent $\mathrm{du}$ succès sous la forme de spectacles prosélytes de lanterne magique ${ }^{41}$, où l'image (r)appelle un commentaire verbal qui lui donne sens. 
[fig. 13]

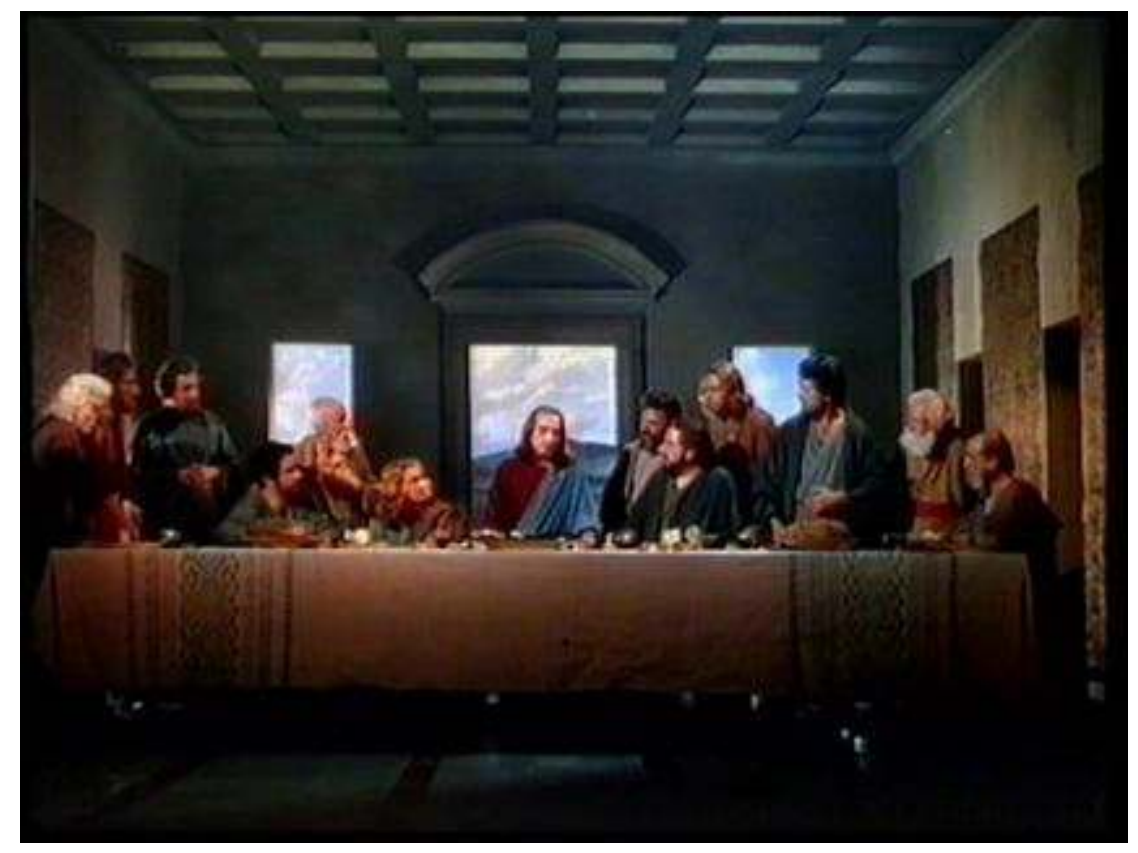

Quo Vadis (Mervyn Le Roy, 1951) [Tous droits réservés]

Ce film de 1951, au moment de représenter le Christ - et plus précisément l'image du Christ transmise aux premiers chrétiens - questionne donc son «dispositif» et son rapport à une tradition visuelle. Renonçant alors à ses potentialités filmiques de mouvement continu narratif et de synchronie sonore, il exploite le plan selon une conception illustrative et tabulaire, réactivant une compréhension didactique de l'image christique comme accompagnement visuel, intermittent et fixe d'un récit verbal. Et si LeRoy reprend la composition de Vinci, c'est en tant qu'«illustration» par excellence, représentation «canonique" de l'événement, ancrée dans l'imaginaire collectif et inscrite dans un "passé » iconographique et technique. Le film ne se "risque " pas à proposer sa propre version du récit catéchétique, il en emprunte le texte, l'image et leur relation à des sources antérieures, validées historiquement et religieusement.

Mais Quo Vadis travaille tout de même cette image différemment d'une illustration fixe. En effet, Pierre énonce ici le dialogue qu'il a échangé avec Jésus en discours direct, et, au moment où il récite les paroles du Christ («This night before the cock crows, thou shall deny me thrice »), l'image redevient "cinématographiquement narrative", si l'on peut dire, puisque Jésus bouge les lèvres dans l'image, sans toutefois que sa voix ne se fasse entendre, «mimant » seulement le discours rapporté par la voix over de Pierre. Et, suite à cette parole, tous les disciples sortent de leur immobilisme pour réagir à cette annonce de trahison en s'animant suivant la composition du tableau de Vinci, qu'ils viennent adopter furtivement. Si dans un premier temps l'image cinématographique est "gelée " en illustration évangélique traditionnelle, le film récupère ensuite la composition comme le moyen d'une mise en mouvement de l'image fixe, et finit donc par inverser le processus d'immobilisation et d'autonomisation $d u$ plan en tableau pour proposer une dynamisation et une linéarisation du tableau en plan. La composition est ainsi rendue « vivante » sous l'action du récit verbal, et par les potentialités narratives du cinéma. 
Le récit tabulaire de la composition de Vinci est en outre réellement linéarisé et modifié par cette inscription dans le mouvement narratif du plan. Les historiens d'art s'accordent en effet généralement à reconnaître dans la gestuelle de la Cène milanaise : la réaction des disciples face à la prophétie du Christ quant à la trahison de l'un d'entre eux ; leur trouble alors que Jésus dénonce Judas; leur révolte quant à l'annonce de la mort prochaine de leur maître ; leur incompréhension devant le mystère de la transsubstantiation ; ou tous ces moments pris dans un «instant prégnant ». Mais il semble qu'aucun commentateur n'ait jamais déchiffré en cette composition la prédiction du reniement de Pierre, qui d'ailleurs est généralement identifié à la droite du Christ, dans le personnage aux cheveux et à la barbe grise - suivant les conventions iconographiques de la figuration de l'apôtre chuchotant, derrière Judas, à l'oreille de Jean (ou de Marie Madeleine...), en tenant un couteau à la main, à l'image de l'épée avec laquelle il voudra défendre le Christ lors de son $\operatorname{arrestation}^{42}$. Si la solution univoque donnée par le film ne résiste pas à une lecture tabulaire analysant le détail, la complexité et la logique symbolique du tableau, elle permet d'inscrire la composition dans une temporalité narrative de cause à effet, conformément à la lecture linéaire propre au plan cinématographique. Le tableau est donc mis ici au service du récit verbal, pour finalement entrer dans le récit filmique, suivant une « transsubstantiation » de l'image et de sa signification.

Mais toutes les citations cinématographiques de la fresque ne se font pas sur le mode sérieux et avec des implications intermédiales intéressantes. Une longue tradition parodique s'est cristallisée autour de cette peinture, et les films ne l'ignorent pas. Un sketch du film History of the world: Part I (Mel Brooks, 1981) trouve ainsi son ressort comique dans le fait de démystifier la si dramatique et si symbolique Sainte Cène par la présence du "serveur " de la tablée, amenant avec lui autant d'éléments et de discours quotidiens et triviaux ${ }^{43}$. La reconstitution de la fresque en tableau vivant sera ainsi parasitée par la présence du serveur incarné par Mel Brooks, et par un plat qu'il tient derrière la tête du Christ, en auréole fortement désacralisée [fig. 14].

[fig. 14]

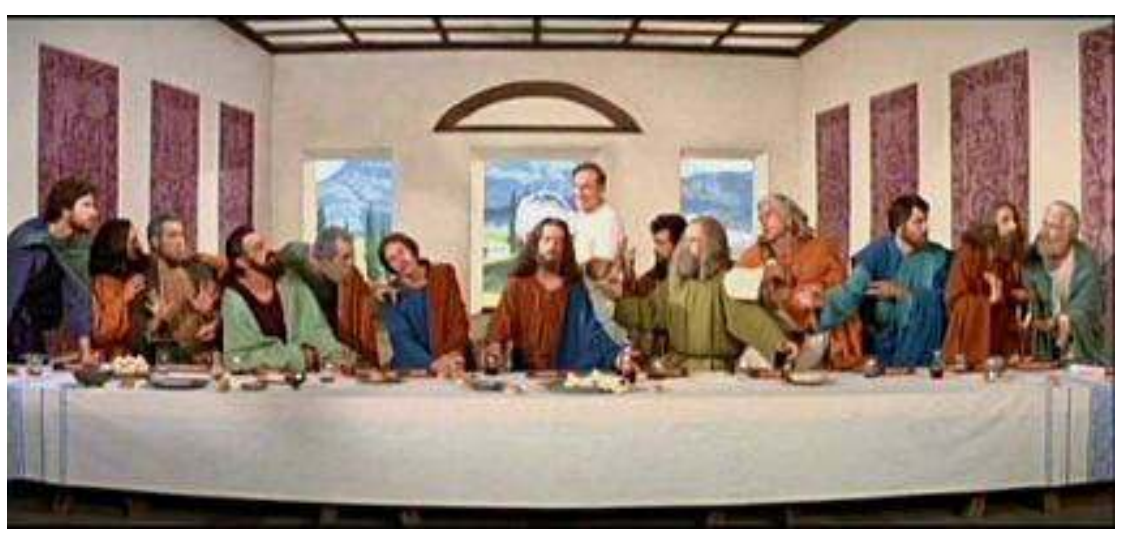

History of the World (Mel brooks, 1981) [Tous droits réservés]

Mais cette version du Cenacolo est surtout intéressante en ce qu'elle se propose de replacer dans une logique narrative, non pas le contenu du tableau mais sa création en tant qu'œuvre. Le film raconte de fait comment Léonard - quatorze siècles avant sa naissance - vient (je cite) «prendre un portrait de groupe ». La pose survient donc en incongruité complète avec la gravité de la cène, présentée comme un absurde caprice des 
saints qui vient désacraliser la «Grande Histoire ». Ainsi la rupture formelle amenée au sein des plans continus par ce subit "plan-tableau» intervient ici pour souligner et exagérer la rupture comique avec le récit.

Enfin, il existe des films qui n'inscrivent pas la Cène léonardesque comme une phase du récit christique mais qui la font apparaître au milieu d'un film consacré à une histoire différente soudain mise en parallèle avec l'Evangile, le plan-tableau survenant comme un déclencheur symbolique faisant " exploser » le sens linéarisé du film.

Les exemples d'un tel usage cinématographique de la composition du Cenacolo, cristallisant une métaphore christique travaillée - de manière plus ou moins anecdotique - dans d'autres plans et séquences du film, sont nombreux. On peut citer M.A.S.H. (Robert Altman, 1970), Il Papocchio (Renzo Arbore, 1980) ou 99 francs (Jan Kounen, 2007), où le surgissement du tableau vivant vise le plus souvent, derrière un " prétexte » symbolique, à ménager une rupture comique au sein de l'enchaînement des plans. Pour que l'allusion à la Sainte Cène soit soutenue par un réel discours artistique, il semble qu'il faille se concentrer sur des films qui, à l'instar de La Ultima Cena (Tomás Guttierez Alea, 1976) ou La dernière (s)cène (Boris Lehman, 1995), consistent tout entiers en une mise en parallèle entre une situation moderne et la situation christique, et font allusion au motif de la cène en général, sans immobiliser la composition en un plan-tableau citant Léonard de Vinci en particulier, mais en renouvelant le sens et la forme à la fois du film et de l'iconographie traditionnelle ${ }^{44}$.

Toutefois, des citations cinématographiques très précises du tableau de Léonard peuvent apparaitre au sein de récits non christiques avec une portée symbolique rayonnant tant sur la narration filmique linéaire que sur celle picturale tabulaire. A titre d'ultime exemple, j'étudierai ici le tableau vivant proposé dans Viridiana (Buñuel, 1961). Ce film raconte la «bonne action » de Viridiana, jeune fille qui sort du couvent en croyant s'être fait violée, et, comme pour « rétablir » sa pureté et sa foi dans les valeurs chrétiennes, met l'immense villa dont elle hérite à la disposition de tous les sans-abri de la ville. Un soir où elle doit sortir, les miséreux organisent un énorme banquet, souillant tout alentour. Et, lors du festin, ils prennent subitement la pose selon le canevas de la Cène de Vinci [fig. 15]. 
[fig. 15]

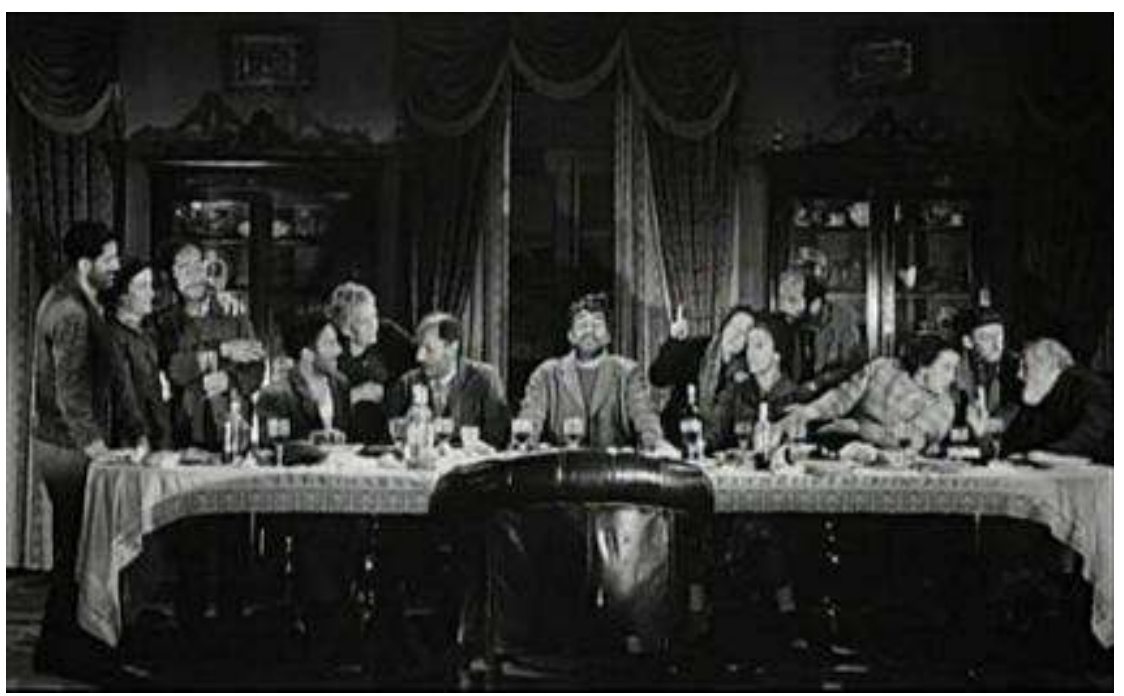

Viridiana (Luis Bunuel, 1961) [Tous droits réservés]

Toute la signification et l'effet comique de cette séquence résident dans l'interférence qu'elle provoque entre les registres «haut» et "bas", noble et trivial, spirituel et corporel. Cette reconstitution soudaine de la composition de Léonard reprend ce qu'il y a de plus valorisé culturellement (et cultuellement), non seulement parce que le tableau est en lui-même un sommet de la "grande » peinture d'histoire (en en incarnant la quintessence à la fois technique et symbolique), mais aussi parce qu'animer soudain la soirée par le spectacle immobile de cette composition, c'est s'adonner à la pratique du tableau vivant tel qu'on l'a définie au XIX ${ }^{\mathrm{e}}$ siècle : en jeu mondain de salon, réservé à une société aristocratique jouant complaisamment de sa propre culture. Dans cette version mise en scène par une société « anti-aristocratique » s'il en est, tout entre en interférence avec ce qu'il y a de plus trivial. Tout dégringole, si l'on peut dire, à commencer par la haute symbolique du tableau, la pose n'ayant d'autre signification ici que de produire une absurde " photo souvenir » et fait immédiatement suite à la révélation d'un mystère, non pas spirituel, mais simplement scabreux : « la gallina [la poule] », solution d'une devinette au double sens grivois venant d'être divulguée au moment où l'ordre de «ne plus bouger » intime aux convives de s'immobiliser.

34 Le ton parodique semble s'éloigner au moment de la pose, puisque le silence s'installe en même temps que le sérieux, et qu'un effet sonore très subtil, consistant dans le chant d'un coq, vient récupérer l'isotopie "gallinacée » de la devinette, non dans un sens grivois mais dans un haut symbolisme convoquant le récit biblique, où le chant du coq intervient après que Pierre a renié le Christ par trois fois, comme un écho et une preuve des paroles prophétiques de trahison énoncées par Jésus durant la Sainte Cène. Introduire le chant du coq dans cette image «muette » et "arrêtée » prend donc un sens profond, permettant métaphoriquement de lui insuffler parole et sens narratif.

Mais l'interprétation scabreuse de l'isotopie aviaire revient et triomphe lorsqu'en guise de prise de vue (ou, comme on dit communément, lorsque « le petit oiseau va sortir»), la " photographe » soulève ses jupes en riant très fort. Au même titre que la signification du tableau en "prenait pour son grade ", sa reconstitution en tableau vivant destitue le jeu mondain érudit en blague grivoise. Notons toutefois que ce basculement réactive quelque 
chose du double régime « haut »-« bas » appartenant historiquement au tableau vivant, dont la «carrière ", comme l'a remarqué Bernard Vouilloux, «ne fut pas exclusivement mondaine", et "ne tarda pas à s'encanailler» de par les "ressources vénales", exhibitionnistes et voyeuristes, de ce spectacle des corps qui fut immédiatement exploité hors des salons, dans un cadre purement érotique ${ }^{45}$.

Ainsi, les effets de sens se multiplient et révèlent l'existence de cette tension entre haut et bas, entre spiritualité et corporalité, à la fois dans le film (où toute la scène joue d'ambivalence), dans le tableau vivant (réactivant à la fois son histoire mondaine et licencieuse) et même dans le tableau lui-même (puisque l'eucharistie instituée par le Christ consiste elle-même à assimiler un acte alimentaire à un acte spirituel).

outre, le traitement formel fait de ce «tableau-vivant » une mise en abyme du film. Le fait de thématiser une pratique de «mise en scène » et de " prise de vue » engage déjà une très forte réflexivité de cette séquence. De plus, la "présence» de l'instance narrative se manifeste en creux dans cette pose de groupe qui est donnée - sans crédibilité aucune - comme purement improvisée par ces pauvres hères, qui ne se disent rien mais créent le tableau ex-nihilo, tous simultanément, sans aucune instruction et dans une fidélité artistique si parfaite que même la trouée de la porte sous la fresque est restituée par le fauteuil ! Enfin ce plan-tableau, qui demeure «pris » dans le film, dans sa logique de montage - en étant « redécoupé » selon différents points de vue et échelles de plan - mais qui néanmoins s'autonomise et s'arrête en tableau, force à une distanciation du spectateur, incité à réfléchir à l'« intégration » de cette scène dans le récit filmique.

Il devra alors constater que la mise en abyme tient ses promesses, et que cette séquence propose un métadiscours sur le film, en en donnant la "clé narrative ». Car à y regarder de plus près, la tension entre " haut » et " bas » qui structurait la séquence traverse tout le film. En effet, jusqu'à cette (s)cène, le film montre Viridiana dans sa volonté de faire triompher la logique chrétienne sur la logique matérielle. Après ce « dernier souper » où les protégés «trahiront» leur "sauveuse» en saccageant la maison et la voie « rédemptrice » qu'elle incarnait, les miséreux s'enfuiront, retourneront à la liberté de la rue et Viridiana abandonnera son voile et ses prières pour livrer son corps au premier venu. C'est donc la logique charnelle qui triomphera. Mais en cet instant de la cène, suspendu et donc selon la signification historique des «tableaux dramatiques" assimilable à un climax de l'œuvre, les deux logiques sont en équilibre, coprésentes, il n'en est pas une qui domine l'autre. Le corporel et le sacré entrent en parfaite interaction, se nourrissant l'un de l'autre pour faire à la fois rire et réfléchir. Cet équilibre précaire, instantané, semble ainsi prôné comme l'impossible "modèle» du film. La stase «tabulaire » de l'image cinématographique est donc travaillée par Buñuel comme un « instant prégnant » de son film, dont elle condense et fait « rayonner » la signification, en écho direct à la concentration sémiotique de la fresque. En se réappropriant l'image, Buñuel se réapproprie ses fonctions symbolico-narratives et les décuple en tous niveaux.

En guise de conclusion, je produis, non une bande cinématographique mais un $\operatorname{objet}^{46}:$ le coffret VHS du film La Vida de Cristo, version doublée en espagnol de The Living Christ Series (John J. Coyle, 1951) où chacune des six cassettes réunit deux épisodes de ce film continu ayant la structure d'un feuilleton. Ce coffret nous intéresse en ce qu'il est décoré à l'effigie de la Cène de Léonard et qu'il est comme une «matérialisation» de la double 
logique du plan-tableau dégagée dans les films montrant le Christ. En effet, dans ce boîtier, de manière concrète et palpable :

- d'une part, les cassettes sont encadrées, immobilisées, dans une reconstitution tridimensionnelle du tableau qui soude ces « parties de film » en une image globale [fig. 16a] ;

- d'autre part, les tranches des cassettes « découpent » réellement la Cène en différents fragments, faisant entrer l'image dans une appréhension linéaire, répétitive et séquentielle [fig. 16b].

[fig. 16a]

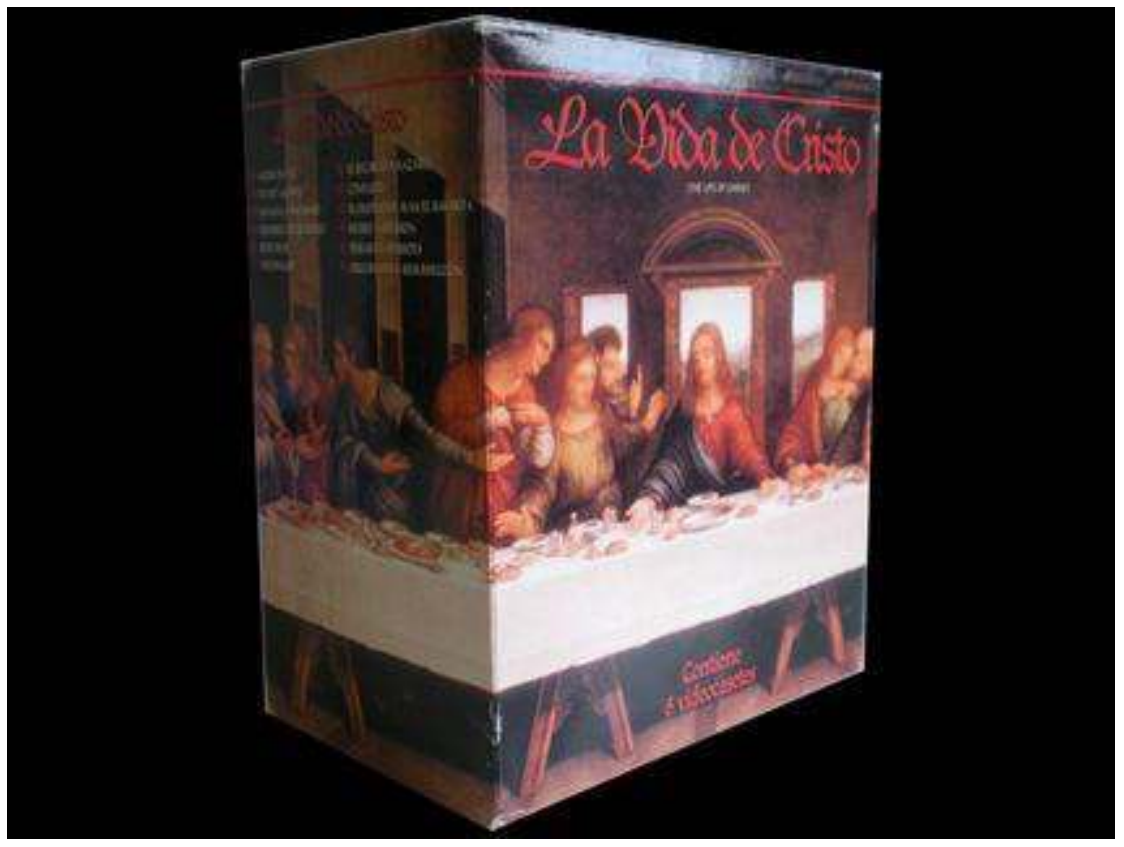

Coffret VHS La Vida de Cristo [Tous droits réservés] 
[fig. 16b]

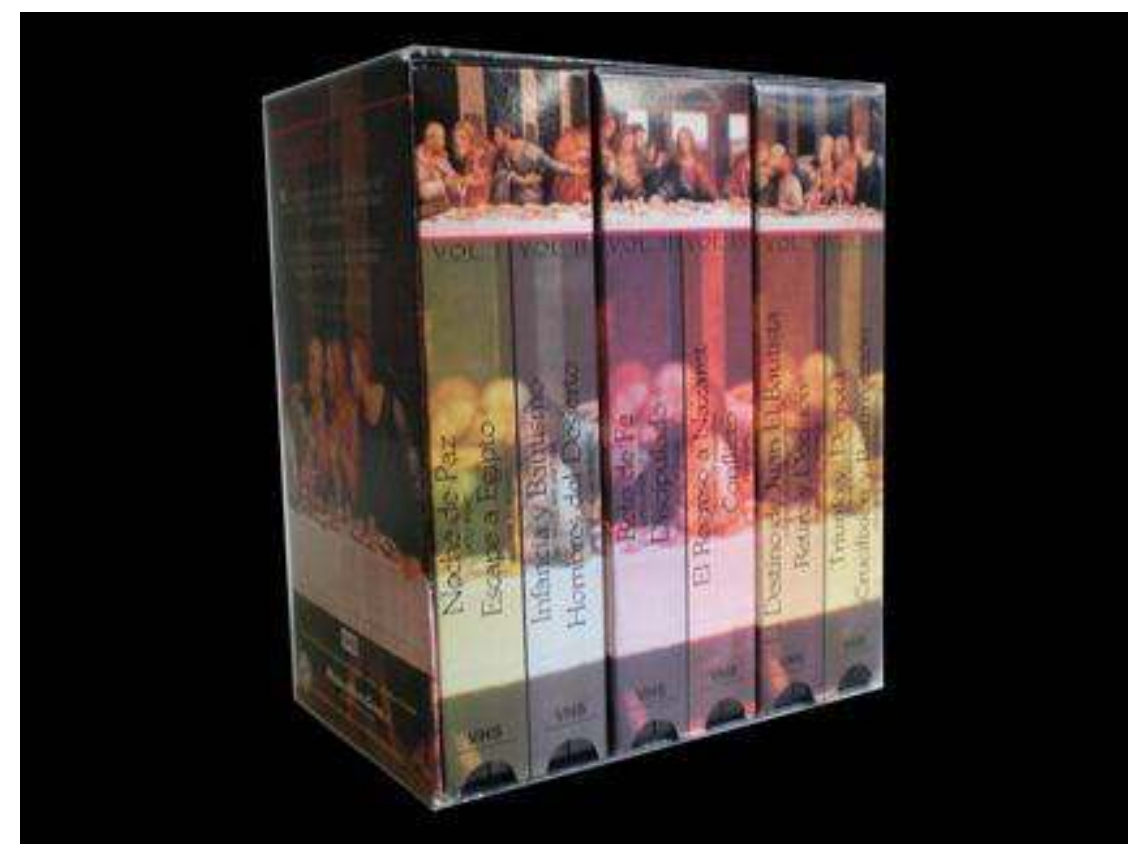

Coffret VHS La Vida de Cristo [Tous droits réservés]

Et lorsqu'on sait que le film ainsi emboîté ne produit au visionnement aucun tableauvivant de la fameuse Cène, on peut interpréter ce geste décoratif à l'aune d'un discours général sur tous les films narratifs se référant à une iconographie christique, postulant que derrière leurs plans résidera toujours un tableau en puissance, l'héritage de cinq siècles de peinture d'histoire, dont l'emblème est incarné par le célébrissime Cenacolo de Léonard. Et si l'interférence avec ces tableaux pourra, comme on l'a vu, être thématisée et cultivée par les plans dans des effets de sens prolongeant le récit cinématographique, ces films évoquant Jésus demeureront toujours, même lorsque la référence iconographique semble ignorée, à la lisière du tableau, à la rencontre des modes tabulaires et linéaires, à la croisée de multiples récits et images.

\section{NOTES}

1. Cet article s'inscrit dans une recherche de thèse de doctorat que je mène à l'Université de Lausanne, Faculté des Lettres sous la direction du Professeur François Albera, intitulée « Le cinéma en tableau vivant »; il est également lié au projet de recherche FNS «Usages de Jésus au XXe siècle » dirigé par Alain Boillat, Jean Kaempfer et Philippe Kaenel.

2. Paul Ricoeur, «Préface ", in : André Gaudreault, Du littéraire au filmique. Système du récit, Paris, Armand Colin, 1999 (1988), p.15.

3. Ibid. Les conclusions narratologiques de ce texte de 1988, réédité en 1999, furent synthétisées par l'auteur en 1990 dans un ouvrage de référence coécrit avec François Jost 
(Le récit cinématographique, Paris, Nathan), lui-même réédité en 2005. Elles marquent ainsi l'un des états les plus accomplis et reconnus de la narratologie filmique.

4. Tom Gunning, « The Non-Continuous Style of Early Films », in : Roger Holman (dir), Cinema 1900-1906 : An Analytical Study, Bruxelles, FIAF, 1982, pp. 219-230.

5. André Bazin, Qu'est-ce que le cinéma, Vol. II, Paris, Cerf, 1959, Chapitre « Peinture et cinéma », pp.127-132. Si l'assimilation de « la » peinture à une dynamique « centripète » et « du » plan filmique à une logique « centrifuge » est nettement caricaturale, cette dichotomie bazinienne me semble pertinente pour décrire les deux « pôles » conceptuels d'une représentation " cadrée », et envisager la tension entre plan et tableau.

6. Jean Giraud, Le Lexique français du cinéma : des origines à 1930, Paris, CNRS, 1958, p.195. 7. Suivant l'autre binôme de la dichotomie proposée par André Bazin, « Peinture et cinéma », op.cit., p. 128 et discutée dans la note 5 . Notons que cette réflexion autour du « cache » filmique par comparaison au « cadre » pictural est amorcée par Bazin en 1956 dans « Un film bergsonien : Le Mystère Picasso », Cahiers du cinéma, n60, juin 1956, pp. 25-28.

8. Il semble que ce soit Pascal Bonitzer, en 1985, dans un article précisément intitulé « Le plan-tableau » (Cahiers du cinéma, n³70, avril 1985, pp. 16-22, réédité en chapitre dans Pascal Bonitzer, Décadrages, Paris, l'Etoile, 1985, pp. 29-41) qui propose le premier cette notion pour définir « le plan qui [...] est pensé, construit, composé comme un tableau ». Si Bonitzer n'évoque aucunement le cinéma des premiers temps et reconnaît le parangon du « plan-tableau » dans des plans de Passion (Godard, 1982), La Ricotta (Pasolini, 1963), La Marquise d'O (Rohmer, 1976) ou L'Hypothèse du tableau volé (Ruiz, 1979) qui imitent délibérément un tableau célèbre via une mise en scène « arrêtée » en tableau vivant, et si Gaudreault n'utilise pas directement le terme de " plan-tableau », Paul Ricoeur, dans sa préface à Du littéraire au filmique, reformule le changement de paradigme décrit par Gaudreault en termes de " passage du plan-tableau au film proprement dit, où le plan s'efface en tant qu'unité autonome » (op.cit., p.15, c'est moi qui souligne). J'opte donc pour ce terme afin de décrire l'élaboration unitaire et « picturalisante » du plan, telle que le cinéma des tout premiers temps, aussi bien que certains cinéastes contemporains, l'ont réalisée - je laisse de côté (ou tout du moins entre guillemets) la terminologie à mon sens trop connotée (historiquement et péjorativement) de Burch qui renomme les plans ainsi conçus des « tableaux primitifs ». (Noël Burch, La Lucarne de l'infini, Paris, Nathan, 1990, chapitre « Passions, poursuites : d'une certaine linéarisation », pp.137-155.)

9. André Gaudreault, op.cit., p.53.

10. Rappelons que l'acception du terme « histoire » (dérivé du terme albertien « istoria ») dans la locution « peinture d'histoire » n'est pas historique mais narrative, désignant une « peinture qui raconte une histoire » et non une « peinture des évènements du passé dignes de mémoire ». Cf. Leon Battista Alberti, De la peinture - De Pictura, Paris, Macula Dédale,1992 (1435), pp. 115 et passim.

11. Cette terminologie d'une lecture « tabulaire » ou « linéaire » de l'image est élaborée par Pierre Fresnault-Desruelles dans le contexte particulier de la bande dessinée, où les cases sont prises dans une même tension entre « tableaux unitaires » et « fragments interdépendants ». Cf. Pierre Fresnault Desruelles, « Du linéaire au tabulaire », Communications, $\mathrm{n}^{\circ} 24,1976$, pp. 7-23.

12. Pour reprendre la terminologie de Burch, qui renomme le changement de paradigme filmique décelé par Gaudreault en termes de « passage » du « mode de représentation primitif $[\mathrm{MRP}] »$ au « mode de représentation institutionnalisé [MRI] ». (Noël Burch, op.cit. , pp. 6-7 et passim.) 
13. J'utilise cette notion de «tableau vivant » dans un sens commun et diachronique - tel qu'il apparait dans les dictionnaires de langue Robert ou Larousse - en tant que pratique consistant à reconstituer, par un groupe de figurants tenant la pose, la composition d'un tableau célèbre. (Je n'entre pas dans une acception historique qui le limiterait à son usage mondain du XIXe siècle et le distinguerait de ses usages antérieurs ou postérieurs, dramatiques, pantomimiques ou cinématographiques.)

14. Sur les origines de ce motif iconographique, voir Marguerite Rassart-Debergh, « Le baptême du Christ dans l'art », Le Monde copte, n¹3, 1988, pp. 17-22.

15. Cette narrativité que Gaudreault a nommée « extrinsèque » a été reconsidérée en termes bel et bien « intrinsèques » mais graduels par Alain Boillat, qui, s'interrogeant sur le rôle du bonimenteur, a rappelé l'hypothèse de Gardies selon lequel « chaque geste ou menu évènement renvoie à tel ou tel passage du récit sacré, qui, en retour, fait de ce[s] film[s] non pas un simple geste monstratif mais une véritable narration ", et posé de manière générale que « l'évènement profilmique induit un degré plus ou moins élevé de narrativité que le monstrateur peut accentuer en fonction du champ couvert par la caméra, du choix des actions filmées, du moment de l'enclenchement des prises de vues, etc. » (Alain Boillat, Du bonimenteur à la voix over. Voix-attraction et voix-narration au cinéma, Lausanne, Antipodes, 2007, pp. 74 et 216) D'ailleurs Noël Burch relativisait déjà partiellement son propos lorsqu'il reformulait le postulat de Gaudreault en expliquant que « les images [des Passions filmées des premiers temps] ne comportent pas, le plus souvent, les indices intrinsèques d'un enchaînement nécessaire ». (Noël Burch, op.cit., p.140, c'est moi qui souligne.)

16. Il est en outre à noter que cette dynamique paradoxale appartient en propre au médium cinématographique, le photogramme étant à la fois mis en mouvement et mis en « arrêt » par la mécanique du projecteur, cette réalité technique étant perceptible au spectateur de la première décennie du siècle, à travers le scintillement de l'image et le travail du projectionniste, libre de ralentir - accélérer - répéter ou arrêter ses tours de manivelles, comme le démontre Timothy Barnard, «L'Opérateur des vues animées : deus ex machina des premières salles de cinéma (suite et fin) », Cinémas, v. 13, n¹-2, 2002, pp. 187-210.

17. Si Alberti écrit : «Et il est bon que dans une histoire il y ait quelqu'un qui avertisse ( admoneat dans le texte en latin (1435) et amnonisca dans le texte en italien (1436)) les spectateurs de ce qui s'y passe ; que de la main il invite à regarder [...] ou que par un visage menaçant ou des yeux farouches, il leur interdise d'approcher, ou qu'il leur indique qu'il y a là un danger ou une chose digne d'admiration, ou encore que, par ses gestes, il t'invite à rire ou à pleurer avec les personnages, enfin [...] [qu'il] concoure à accomplir et enseigner l'histoire. » (op.cit., p.179), c'est Alain Laframboise qui propose de nommer en français ce personnage l'« admoniteur » dans Istoria et théorie de l'art. Italie XVe et XVIe siècles, Montreal, PUM, 1989 (Tandis que John Spencer, qui a traduit le texte d'Alberti en anglais en 1966 [Leon Battista Alberti. On Painting, London, Yale University Press] parle de « commentator » et est à l'origine de la locution française concurrente de « commentateur albertien »).

18. André Chaperon, « Le hors-champ de l'histoire. Une lecture benjaminienne de Tom, Tom, The Piper's Son », Décadrages. Cinéma à travers champs, n¹-2, 2003, pp. 17-34.

19. Selon Alberti qui définit le « mouvement de l'âme » produit par un tableau comme la « contagion » du sentiment représenté, les figures imagées ayant comme « pouvoir » de transmettre au spectateur leur état d'âme. Op. cit, p. 175. 
20. «Tableau dramatique » est la locution que Bernard Vouilloux utilise et dit reprendre à Diderot dans son ouvrage Le Tableau vivant. Phryné, l'orateur et le peintre, Paris, Flammarion, 2002, pp. 26-27 et passim. Dans l'historiographie du théâtre du XVIII ${ }^{\mathrm{e}}$ et du $\mathrm{XIX}^{\mathrm{e}}$ siècles, cet usage du tableau vivant a néanmoins connu d'autres terminologies, en ayant également été appelé " tableau-comble », « tableau-stase », « tableaux fugitifs » ou encore « tableaux mis en action ».

21. L'exemple par excellence en est la Trinité peinte par Masaccio en 1428, où le regard frontal accusateur et le geste dénonciateur de la Vierge intime avec violence le spectateur à une prise de conscience, et l'enjoint par la menace au chemin de la rédemption dont on comprend toutes les étapes (le renoncement à la chair, la piété, l'intercession des saints, la rédemption christique, la foi en la trinité) en suivant le parcours ascensionnel du regard indiqué par la logique signifiante tabulaire de la fresque.

22. La séquence suivante, qui représente la résurrection de façon très métonymique, pouvant être considérée comme l'épilogue du film.

23. Une section de l'exposition The Mind of Leonardo. The Universal Genius at Work, présentée à la Galerie des Offices à Florence du 28 mars 2006 au 7 janvier 2007, proposait un choix d'extraits de films qui reprenaient, de manière plus ou moins exacte, le canevas du fameux Cenacolo. Ces extraits peuvent encore être visionnés sur le site de l'exposition http://brunelleschi.imss.fi.it/menteleonardo/evideo.asp?c=14074\&nf=V_8_B_c\&r=w, tandis qu'Alain Boillat en a proposé un commentaire dans son article « La Cène de Vinci et le cinéma : notes sur une section de l'exposition The Mind of Leonardo aux Offices », paru sur http://www.unil.ch/webdav/site/usagesdejesus/shared/Antamoro_aux_Offices.pdf. 24. Leo Steinberg, Leonardo's Incessant « Last Supper », New York, Zone Books, 2001, p.12. Soulignons la mention précisant la nature fondamentalement « narrative » de cette image (en anglais « narrative picture »).

25. Le plus souvent attribuée à Giovanni Pietro da Birago et renommée « Cène avec un épagneul » pour mettre l'accent sur les ajouts de la gravure, qui en plus d'un chien au bas à droite enrichit la composition d'un phylactère citant les paroles du Christ « amen dico vobis quia un[us] vestrum me traditurus $e$ [st] », autant d'éléments qui linéarisent la signification de la composition.

26. L'image, célèbre, a suscité une plainte pénale émanant du haut-clergé catholique ; l'arrêt du tribunal peut être consulté sur http://www.legifrance.gouv.fr/ affichJuriJudi.do?idTexte=JURITEXT000007055276.

27. Cf. http://www.religioustattoos.net/Other_Tattoos/Last_Supper/index.php

28. Si Dan Savage sur http://slog.thestranger.com/2007/09/

other_last_suppers_wheres_the_outrage ou Laura Sweet dans « The Last Supper. And the one after that. And after that. And then, even more » sur http://

ifitshipitshere.blogspot.com/2008/05/last-supper-and-one-after-that-and.html proposent un premier tour d'horizon (notamment, pour la dernière, dans le domaine des arts graphiques), c'est sûrement la collection compilée par Martin Bregenzer dans le CDROM Zu Tisch, bitte! Rund 150 Bildvariationen zu Leonardo da Vincis « Abendmahl » (c) Calwer Verlag, 2006, qui est la plus complète au mois de juillet 2008.

29. Telles que les panneaux photographiques de Rauf Mamedov (Last Supper, 1997) ou de Renée Cox (Yo Mama's Last Supper, 1996) dont les implications ont été analysées par Nathalie Dietschy, «L'autoportrait en Christ de Renee Cox : étude d'un scandale ", in Jean Kaempfer et alii (ed.), Points de vue sur Jésus au XXe siècle. A la croisée des disciplines, Etudes de Lettres $n^{\circ} 2 / 2008$, pp. 145-168. 
30. Telles que les installations de Devorah Sperber (After the Last Supper, 2005) et Francine LeClercq (sans titre, 2007) qui jouent de cet acquis visuel léonardesque en questionnant et renouvelant le regard du spectateur à l'égard de son Cenacolo.

31. Célèbre roman de Dan Brown publié à New York, Doubleday, 2001.

32. Alain Boillat, « Notes sur une section de l'exposition... », op.cit, p. 4. Cet article propose de fait une analyse approfondie de la séquence de Sainte Cène du film Christus, en soulignant combien le traitement du surnaturel permis par les surimpressions cinématographique vise à renouveler l'iconographie traditionnelle.

33. Léo Steinberg (qui par ailleurs produit le bilan historiographique le plus complet des différentes lectures du tableau, op.cit., chapitre « The Subject », pp. 31-53) propose (pp. 51-53) une interprétation qui nous intéresse tout particulièrement, en ce qu'elle distingue deux « axes » de développement du tableau, tabulaire et linéaire, porteur chacun d'un récit différent. Selon lui le déroulement latéral du tableau, dessiné par la composition des figures, raconte la réaction des apôtres à l'annonce de la trahison de l'un d'eux en en explorant tous les ressorts dramatiques, tandis que l'axe orthogonal « embrassé » par le Christ et s'étendant jusqu'au spectateur par les lignes de projection perspectives, présente le récit de la communion en faisant rayonner ses enjeux symboliques. Précisons que la notion d' ' instant prégnant » est définie par Lessing dans le Laocoon (1766) et que l'historiographie cinématographique a systématisé son opposition avec la notion d'« instant quelconque », concept bergsonien qui a été relu par Gilles Deleuze à l'aune du plan filmique (L'Image Mouvement, 1983).

34. Ce que Vouilloux appelle le "tableau dramatique », Pierre Frantz le nomme en effet « tableau-comble », dans L'esthétique du tableau dans le théâtre du XVIIIe siècle, Paris : Puf, 1998, p. 167 et passim.

35. « [Les] tableaux vivants ne conquièrent l'autonomie formelle [...] qu'après s'être émancipés du cadre dramatique où ils sont d'abord apparus et où ils se confondaient peu ou prou avec le tableau dramatique de Diderot. » Bernard Vouilloux, op.cit., p. 23.

36. Alain Masson, « La toile et l'écran », Positif, nº 189, 1977, p.21.

37. Cf. Mario Carniani (dir), I cenacoli fiorentini : un suggestivo percorso turistico in una Firenze da riscoprire, Firenze, Accoglienza e Promozione Turistica, s.d.

38. Sur cette convention consistant à rejeter le Christ hors-champ, sa « légifération » par la censure anglaise de 1913 à 1961 et la manière dont les films ont cultivé (internationalement et même après 1961, suscitant presque un genre filmique nommé « indirekter Jesusfilm » par Georg Langenhorst) cette « esthétique du point de vue » iconographiquement " décalée », voir mon article « Regards croisés sur la crucifixion : les points de vue du cinéma ", Etudes de Lettres, op.cit., pp. 41-44.

39. Citation de Norman Jewison dans le «Commentaire du film par le réalisateur et l'acteur Ted Neeley » de l'édition DVD @ Universal Studio, 2004.

40. Comme la « méthode Bernadette » citée par Alain Boillat dans « Quand les vignettes prolongent les versets. La bande dessinée en quête du Jésus historique », in Bande dessinée, histoire et sciences humaines, Genève, L'Equinoxe (à paraître).

41. Voir Isabelle Saint-Martin, « Du vitrail à la lanterne magique : le catéchisme en images ", in Lanterne magique, tableaux transparents, Paris, Réunion des Musées nationaux, 1995, pp. 105-120.

42. Le meilleur bilan historiographique des interprétations quant à la signification de la composition est sans nul doute celui proposé par Léo Steinberg, dont l'entièreté de l'ouvrage (op.cit.) « fait le point » sur les lectures du tableau. 
43. Cette séquence, qui joue en outre d'autres effets de décontextualisation comme le quiproquo et l'anachronisme, est disponible en ligne sur http://www.youtube.com/ watch?v=VA1sx-vyWVk\&amp.

44. Il y a évidemment des films « intermédiaires ", comme Mamma Roma (Pier Paolo Pasolini, 1962), où l'allusion iconographique à la cène en général (et non en particulier à celle de Léonard), sans se figer en tableau-vivant (malgré des fondus enchaînés plan-surplan qui ont un effet de dilatation temporelle) et sans être au centre même du film, engage une isotopie iconographique et symbolique très travaillée - comme souvent chez Pasolini - autour du « Fils », du sacrifice, de la condamnation, du renversement de la justice chrétienne, etc.

45. Bernard Vouilloux, op.cit., p. 27.

46. Quoique l'exercice filmique dirigé par Eisenstein (alors qu'il enseignait à l'Institut de cinématographie de Moscou ) et réalisé par son élève Konstantin Pipinasvili en 1934, consistant en une « cinématisation » de la Cène de Léonard doive également être cité et pourrait aussi bien servir de conclusion à ma démonstration (mais je n'en connais que la description textuelle et la mosaïque « délinéarisée » des plans produites dans Edoardo G. Grossi, « Pittura come cinema : La 'cinematizzazione' dell'Ultima Cena di Leonardo », in : Sergej Ejsenstejn : Oltre il cinema [Pietro Montani ed.], Venise, La Biennale di Venezia, 1991, pp. 201-216.) Le principe même de ce film consistait en effet à transformer le tableau de Vinci (ou plus précisément une copie gravée du tableau) en une suite de plans, à changer la logique tabulaire de la peinture/gravure par la découpe et la « concaténation » linéaire du cinéma. Or cette « mise en plans » du tableau tend vers une narrativisation causale de l'image, comme le souligne Grossi (qui explique que la consigne du travail consiste à «traduire (riesprimare) l'action dramatique représentée dans le tableau », p. 207) et comme l'exemplifieront les films sur l'art qui vont se multiplier en Europe autour des années 1940, qui suivront le même principe et connaîtront avec, notamment, Luciano Emmer une portée essentiellement narrative, comme l'annoonce de manière exemplaire le titre de son film sur les fresques de Giotto réalisé en 1938 : « Racconto da un'affresco ».

\section{RÉSUMÉS}

Partant de l'hypothèse qu'au cinéma, un plan travaillé à la manière d'un tableau engage, non pas un «arrêt » du récit, mais un enrichissement de la logique narrative linéaire du film par une sémiotique tabulaire et des jeux de références intermédiales, cet article analyse le cas exemplaire et récurrent des plans qui reconstituent en «tableau vivant" La Cène de Léonard de Vinci. Christus (Giulio Antamoro, 1914-1916), Ben Hur, a tale of the Christ (Fred Niblo, 1925) ou Quo Vadis (Mervyn LeRoy, 1951) y sont explorés, ainsi que d'autres productions qui replacent la composition du Cenacolo à la fois en une "phase » et une "stase " dramatique et esthétique du récit christique. Des films tels que Viridiana (Luis Buñuel, 1961) sont aussi évoqués, qui font apparaître la composition comme une pause allégorique d'un récit contemporain, enrichi sur un plan énonciatif, symbolique et iconographique.

Based on the hypothesis that in the cinema, a shot worked in the style of a picture doesn't "stop" the storyline but expands the linear narrative with tabular semiotics and artistic references, this 
article analyzes the exemplary and recurring case of the shots which reconstitute Leonardo da Vinci's Last Supper as a "living picture". Christus (Giulio Antamoro, 1914-1916), Ben Hur, a tale of the Christ (Fred Niblo, 1925), Quo Vadis (Mervyn LeRoy, 1951) and other movies are investigated, that replace the famous Cenacolo as both a phase and a stasis of the Christlike narrative. Films like Viridiana (Luis Buñuel, 1961) are mentioned too, where da Vinci's composition occurs like an allegorical extension of a contemporary story, enriched both symbolically and iconographically.

\section{INDEX}

Mots-clés : Cène, Christ, cinéma, iconographie, Léonard de Vinci, linéaire, Passion, peinture, plan, tableau, tabulaire

\section{AUTEUR}

\section{VALENTINE ROBERT}

Assistante diplômée en Histoire et esthétique du cinéma à la Faculté des Lettres de l'Université de Lausanne, Valentine Robert prépare actuellement, sous la direction du professeur François Albera, une thèse de doctorat portant sur le tableau vivant au cinéma, ses enjeux en termes de conception de l'image filmique et de relation entre plan cinématographique et tableau scénique ou pictural. Elle est d'autre part associée à une recherche interdisciplinaire du FNS (Fonds National Suisse pour la recherche scientifique), dirigée par Alain Boillat, Jean Kaempfer et Philippe Kaenel, consacrée au destin fictionnel et visuel de la figure du Christ au XXe siècle (voir www.unil.ch/usagesdejesus). 